Supporting Information

\title{
Mononuclear Manganese(III) Superoxo Complexes: Synthesis, Characterization and Reactivity
}

Yen-Hao Lin, ${ }^{\dagger}$ Hanna Hinrika Cramer, ${ }^{\ddagger}$ Maurice van Gastel,${ }^{\perp}$ Yi-Hsuan Tsai,${ }^{\dagger}$ Chi-Yi Chu,${ }^{\dagger}$ Ting-Shen Kuo, ${ }^{\#}$ I-Ren Lee, ${ }^{* \dagger}$ Shengfa Ye, ${ }^{* \perp}$ Eckhard Bill, ${ }^{*}+$ and Way-Zen Lee ${ }^{* \dagger}$

${ }^{\dagger}$ Department of Chemistry, National Taiwan Normal University, Taipei 11677, Taiwan

*Department of Molecular Theory and Spectroscopy, Max-Planck-Institut für Chemische Energiekonversion, Mülheim an der Ruhr D-45470, Germany

${ }^{\perp}$ Department of Molecular Theory and Spectroscopy, Max-Planck-Institut für

Kohlenforschung, Mülheim an der Ruhr D-45470, Germany

\#Instrumentation Center, Department of Chemistry, National Taiwan Normal University, Taipei 11677, Taiwan

*Corresponding author. Email: wzlee@ ntnu.edu.tw (W.-Z.L); shengfa.ye@kofo.mpg.de (S.Y.); eckhard.bill@cec.mpg.de (E.B.); irenlee@ntnu.edu.tw (I.-R.L)

Contents

Figures S1-S18

Tables S1-S5

References 1-16 

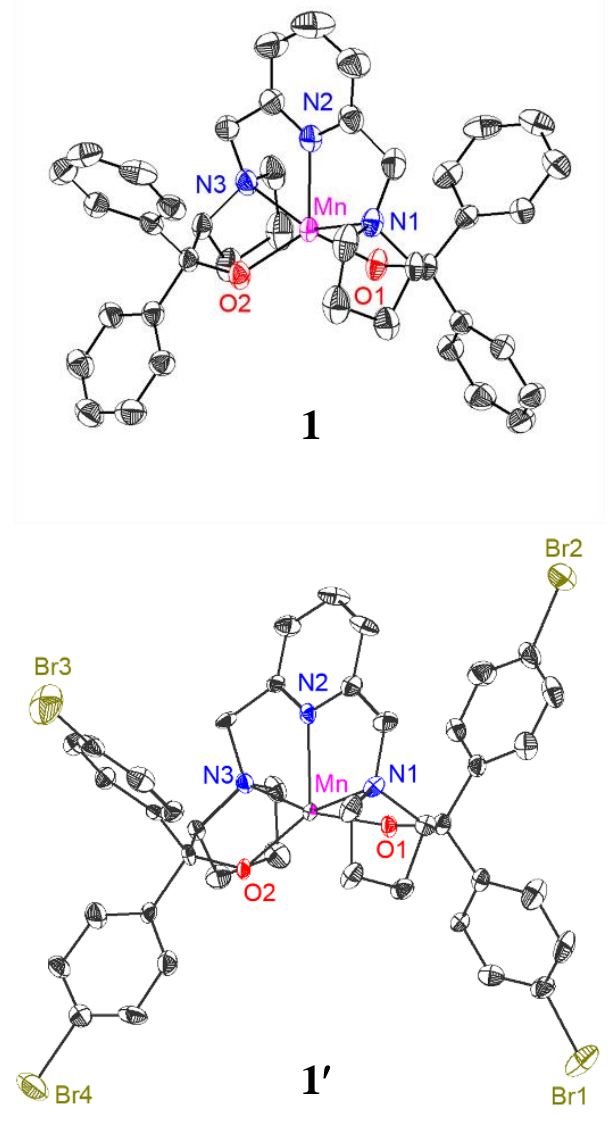

Figure S1. ORTEP presentation of $\mathrm{Mn}(\mathrm{BDPP})(\mathbf{1})$ and $\mathrm{Mn}\left(\mathrm{BDP}^{\mathrm{Br}} \mathrm{P}\right)\left(\mathbf{1}^{\prime}\right)$. Hydrogen atoms are omitted for clarity. 


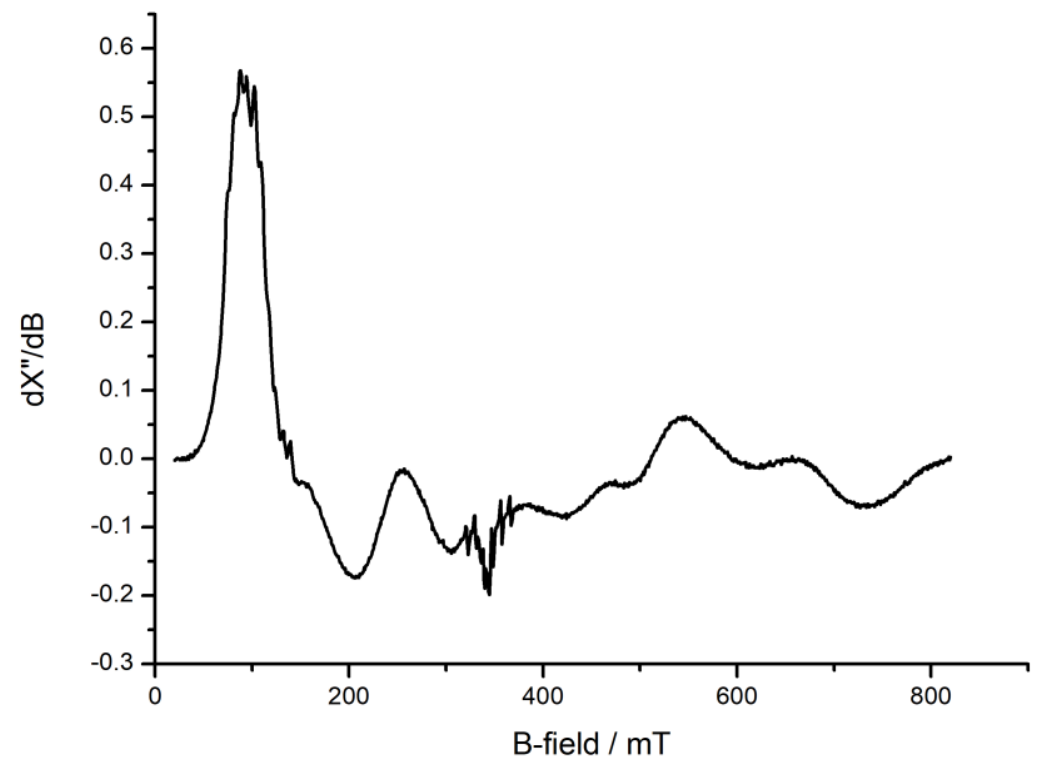

Figure S2. EPR spectrum of $1(1 \mathrm{mM})$ in MeTHF recorded at $10 \mathrm{~K}$. (Frequency $9.65629 \mathrm{GHz}$, power $0.2 \mathrm{~mW}$, modulation $100 \mathrm{kHz} / 0.75 \mathrm{mT}$ )

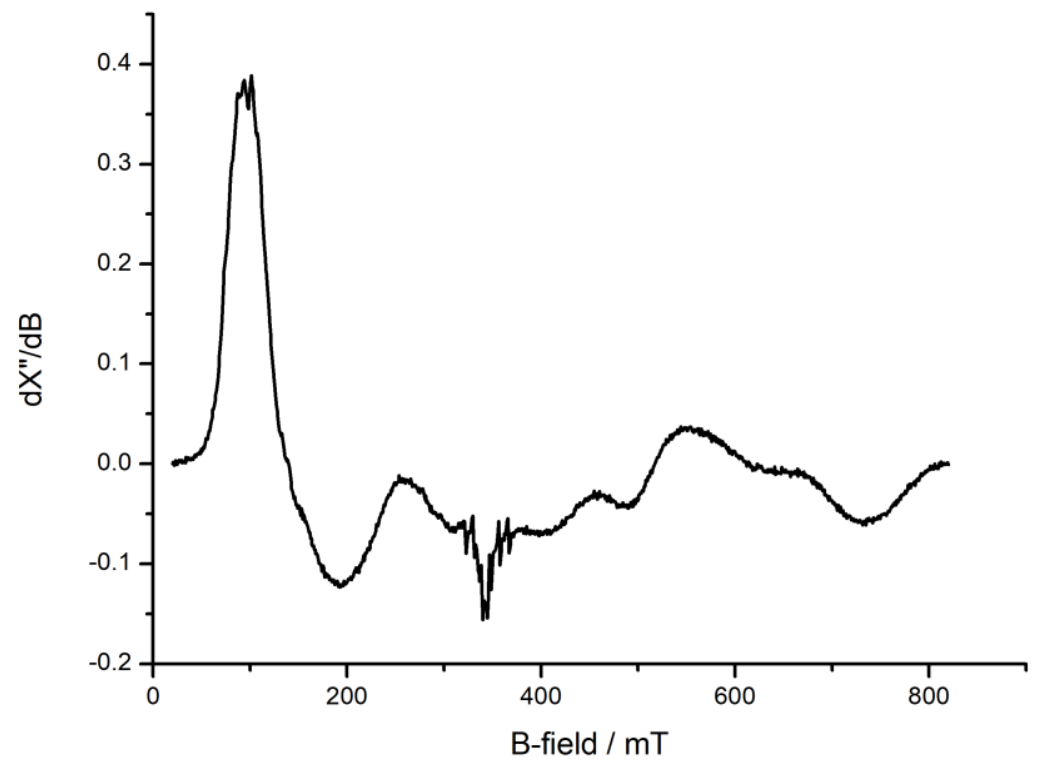

Figure S3. EPR spectrum of 1' (1 mM) in MeTHF recorded at $10 \mathrm{~K}$. (Frequency $9.65864 \mathrm{GHz}$, power $0.2 \mathrm{~mW}$, modulation $100 \mathrm{kHz} / 0.75 \mathrm{mT}$ ) 


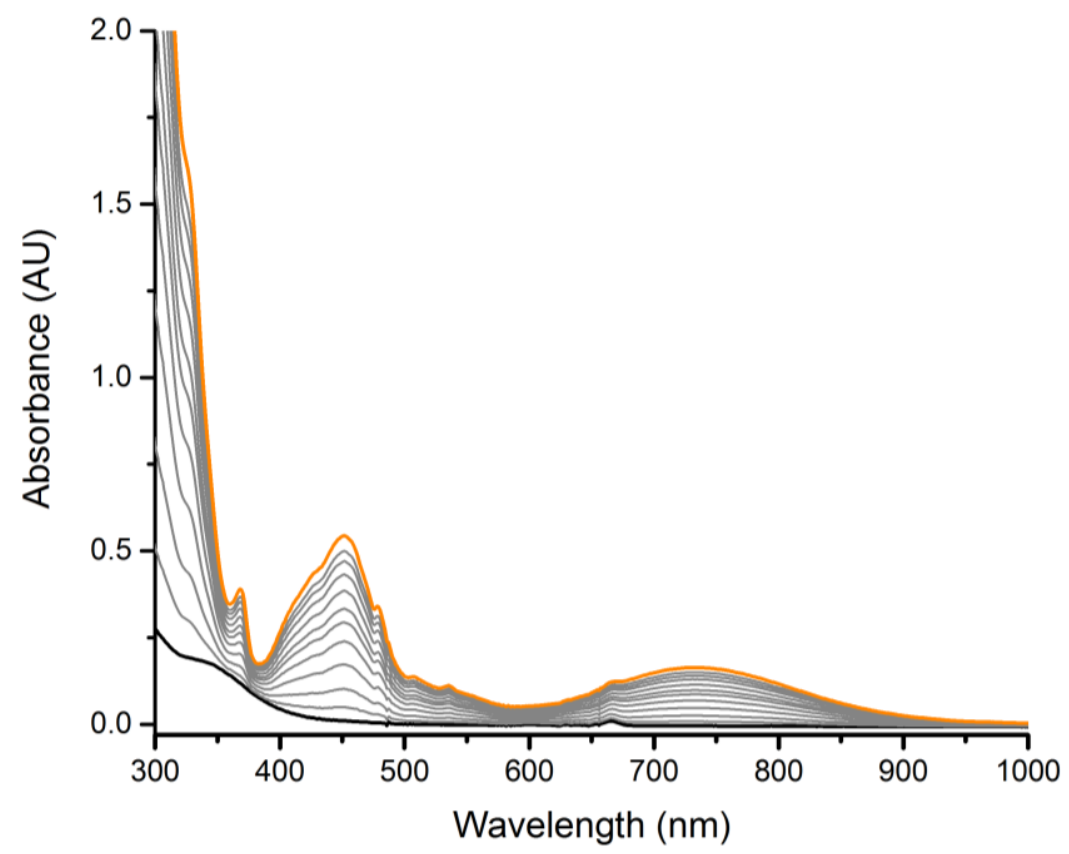

Figure S4. UV-vis spectra of 1 (black, $0.4 \mathrm{mM}$ ) reacting with $\mathrm{O}_{2}$ forming 2 (orange, $0.4 \mathrm{mM}$ ) in $\mathrm{THF}$ at $-80^{\circ} \mathrm{C}$. 
(a)

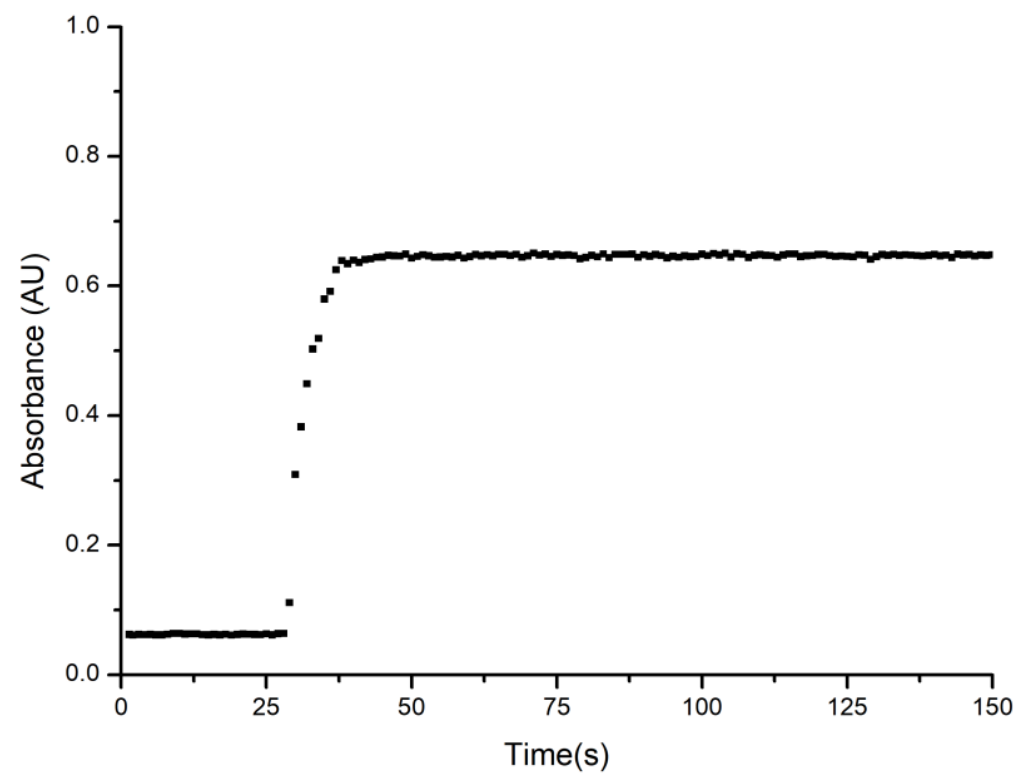

(b)

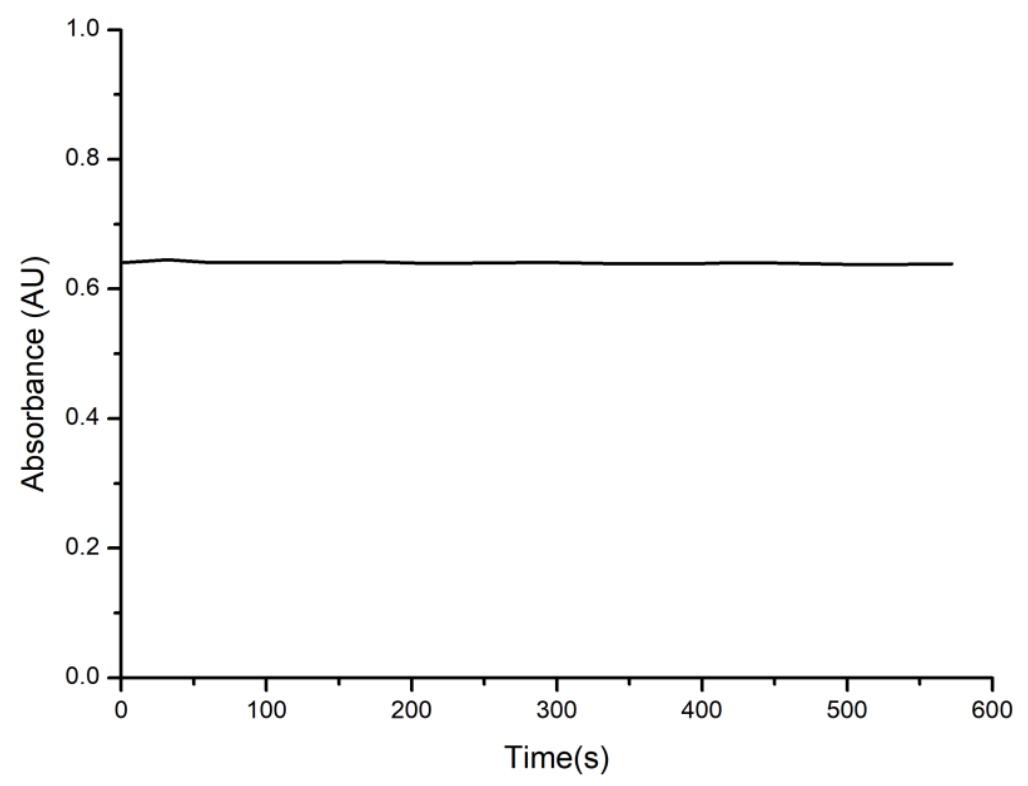

Figure S5. (a) The trace of formation of $\mathbf{2}^{\prime}(0.4 \mathrm{mM})$ by bubbling $\mathrm{O}_{2}$ into a THF solution of $\mathbf{1}$ from the monitoring for the absorption band at $450 \mathrm{~nm}$; (b) The trace of retention of $\mathbf{2}^{\prime}$ in THF $(0.4 \mathrm{mM})$ from purging the solution with argon for $10 \mathrm{mins}$ at $-80{ }^{\circ} \mathrm{C}$. 


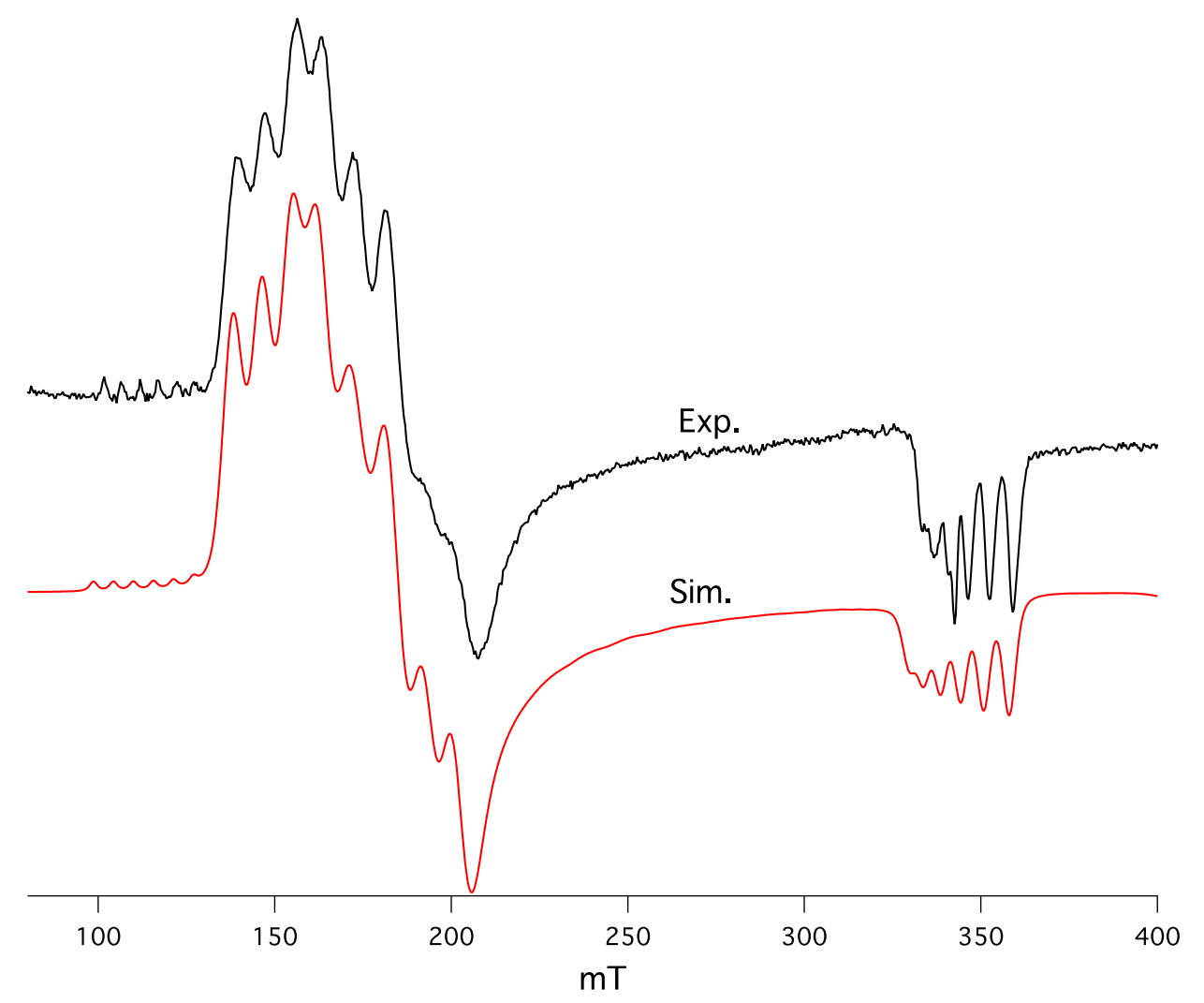

Figure S6. EPR spectrum of $2(1 \mathrm{mM})$ recorded at $10 \mathrm{~K}$. (Frequency $9.655 \mathrm{GHz}$, power 0.2 $\mathrm{mW}$, modulation $100 \mathrm{kHz} / 0.75 \mathrm{mT})$. The simulation was obtained by the following parameter, $g_{\text {iso }}=1.997(10), D=-5.4(5) \mathrm{cm}^{-1}, E / D=0.05(1)$, and $\left|A_{\mathrm{x}, \mathrm{y}, \mathrm{z}}\right|=237(10), 223(10), 158(10) \mathrm{MHz}$. The spin concentration of the superoxo complex with $S=3 / 2$ was determined two different samples to be ca. 95 and $99 \%$ of the chemical concentration $(2 \mathrm{mM})$, with ca. $\pm 10 \%$ error. To this end, the prominent subspectrum from the 'allowed' transitions within the excited Kramers doublet $\left(\mathrm{m}_{\mathrm{s}}= \pm 1 / 2\right)$ with effective $\mathrm{g}$ values at 4 and 2 was numerically double-integrated in the field range $130-380 \mathrm{mT}$, and compared with the integrated spectrum of a $1 \mathrm{mM} \mathrm{Cu}$ (II) standard measured at non-saturating conditions $(30 \mathrm{~K}, 0.05 \mathrm{~mW})$. Both spectra were corrected for different Aasa-Vanngard factors for field-swept spectra, arising from different (effective) $g$ values. ${ }^{1}$ In addition, the $m_{s}= \pm 1 / 2$ subspectrum of the target compound 2 was corrected for the corresponding Boltzmann factor of the excited state by using $D=-5.2 \mathrm{~cm}^{-1}$ taken form the analysis of the temperature variation described in the main text. 


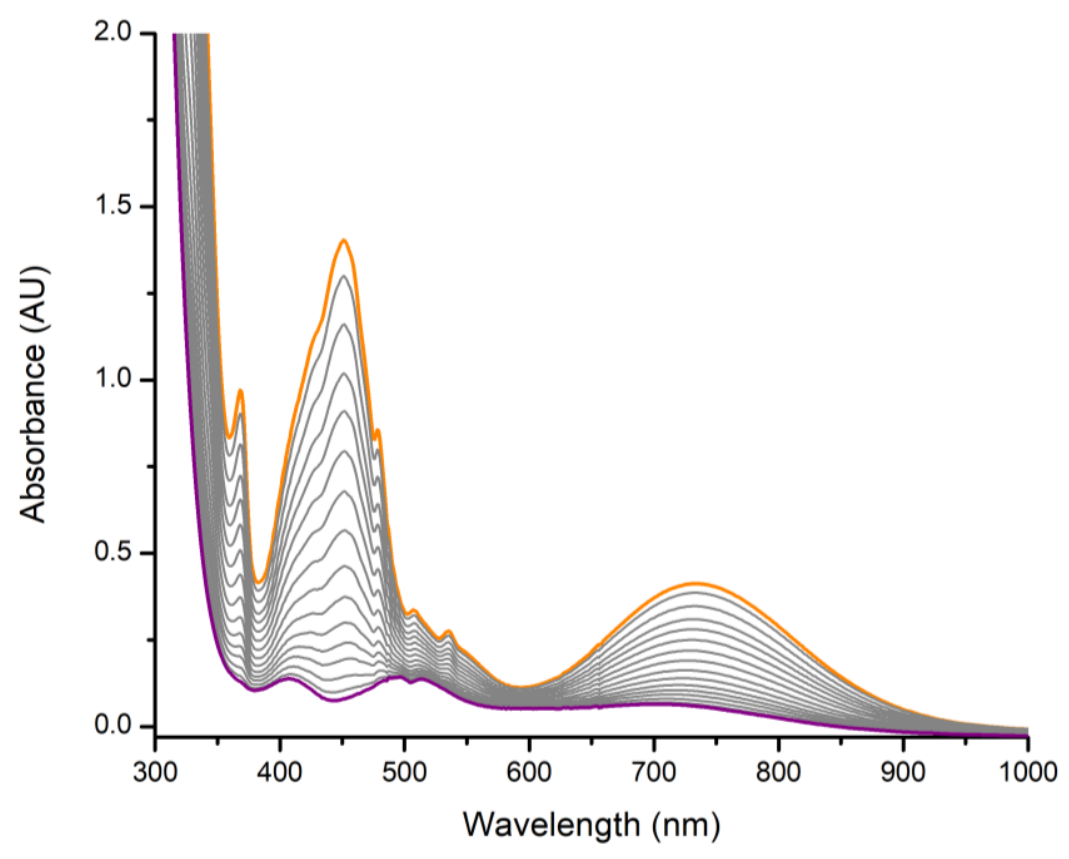

Figure S7. UV-vis spectra of 2 (orange line, $1 \mathrm{mM}$ ) reacting with 10 equivalents of TEMPOH in THF at $-90^{\circ} \mathrm{C}$. Purple line represents $\mathrm{Mn}^{\mathrm{III}}(\mathrm{BDPP})(\mathrm{OOH})(\mathbf{5})$ with four absorption bands at $410,495,515$ and $720 \mathrm{~nm}$. 
(a)

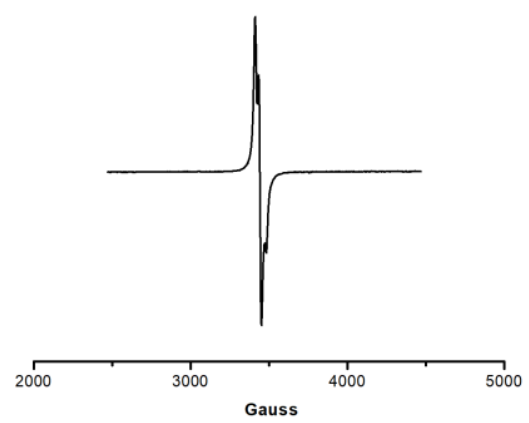

(b)

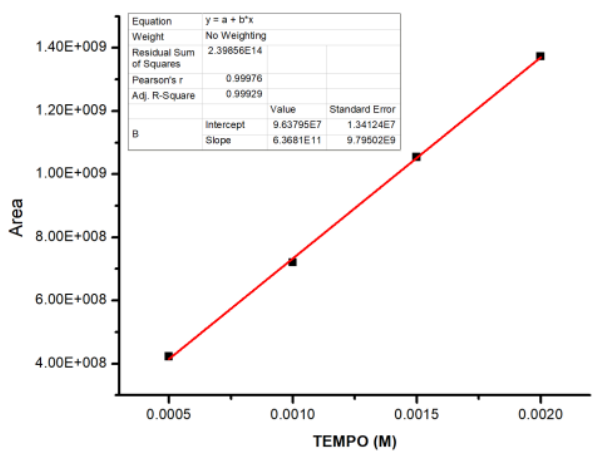

Figure S8. (a) EPR signal $(\mathrm{g}=2.006,77 \mathrm{~K})$ of the produced TEMPO radical (integrated area: $6.5 \times 10^{8}, 86 \%$ vs calibration) obtained from the reaction of $2\left(1.0 \times 10^{-3} \mathrm{M}\right)$ with 10 equivalents of TEMPOH at $-90{ }^{\circ} \mathrm{C}$; (b) the calibration curve of TEMPO radical.

(a)

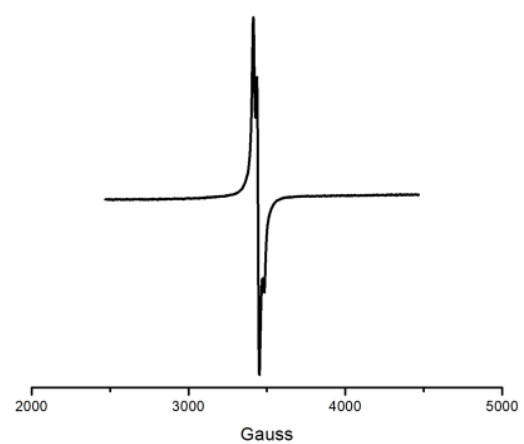

(b)

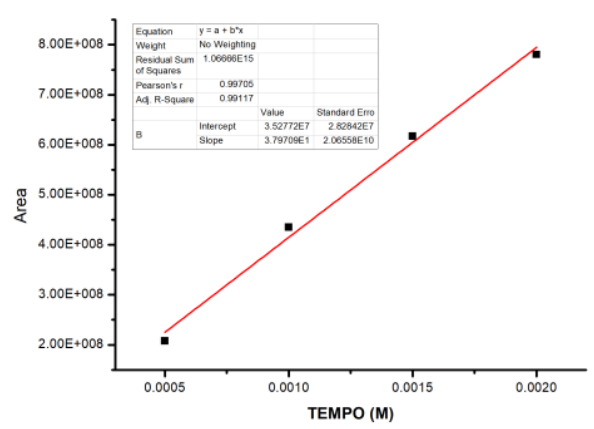

Figure S9. (a) EPR signal $(\mathrm{g}=2.006,77 \mathrm{~K})$ of the produced TEMPO radical (integrated area: $3.8 \times 10^{8}, 93 \%$ vs calibration) obtained from the reaction of $\mathbf{2}^{\prime}\left(1.0 \times 10^{-3} \mathrm{M}\right)$ with 10 equivalents of TEMPOH at $-90{ }^{\circ} \mathrm{C}$; (b) the calibration curve of TEMPO radical. 


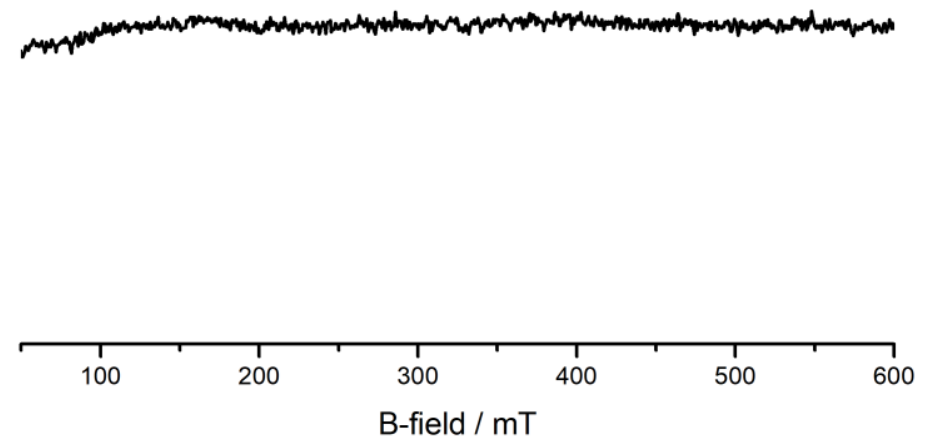

Figure S10. EPR spectrum of 6' $(1 \mathrm{mM})$ recorded at $10 \mathrm{~K}$. (Frequency $9.388 \mathrm{GHz}$, power 0.2 $\mathrm{mW}$, modulation $100 \mathrm{kHz} / 0.75 \mathrm{mT})$. 
(a)

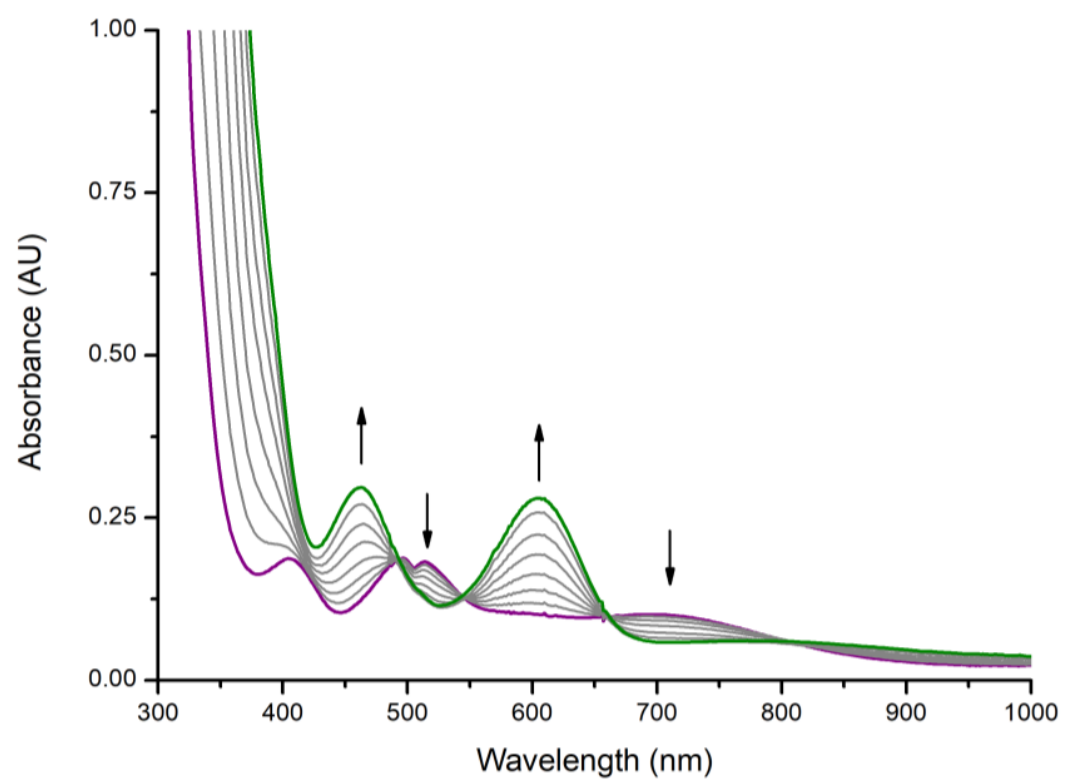

(b)

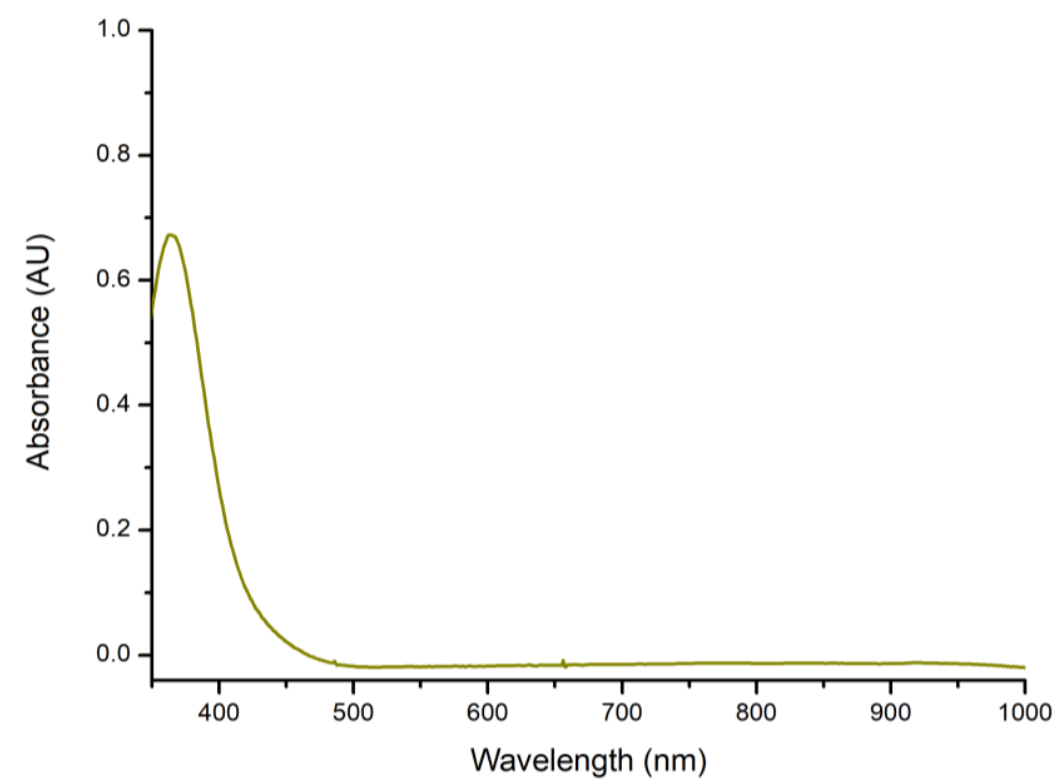

Figure S11. (a) Reaction of 5 with one equiv of HOTf in THF forming [Mn(BDPP)( $\left.\left.\mathrm{H}_{2} \mathrm{O}\right)\right] \mathrm{OTf}$ (6), which was subsequently warmed up. (b) $100 \mu \mathrm{L}$ of the resulting solution was added into an acetone solution of excess $\mathrm{NaI}$ to examine the yield of the produced $\mathrm{H}_{2} \mathrm{O}_{2}$. The theoretical concentration of the in situ generated $\mathrm{I}_{3}{ }^{-}$solution is $3.2 \times 10^{-5} \mathrm{M}$. The amount of the formed $\mathrm{I}_{3}^{-}$was then quantified using its visible spectrum $\left(\lambda_{\max }=361 \mathrm{~nm}, \varepsilon=2.5 \times 10^{4} \mathrm{M}^{-1} \mathrm{~cm}^{-1}\right)$. 
(a)

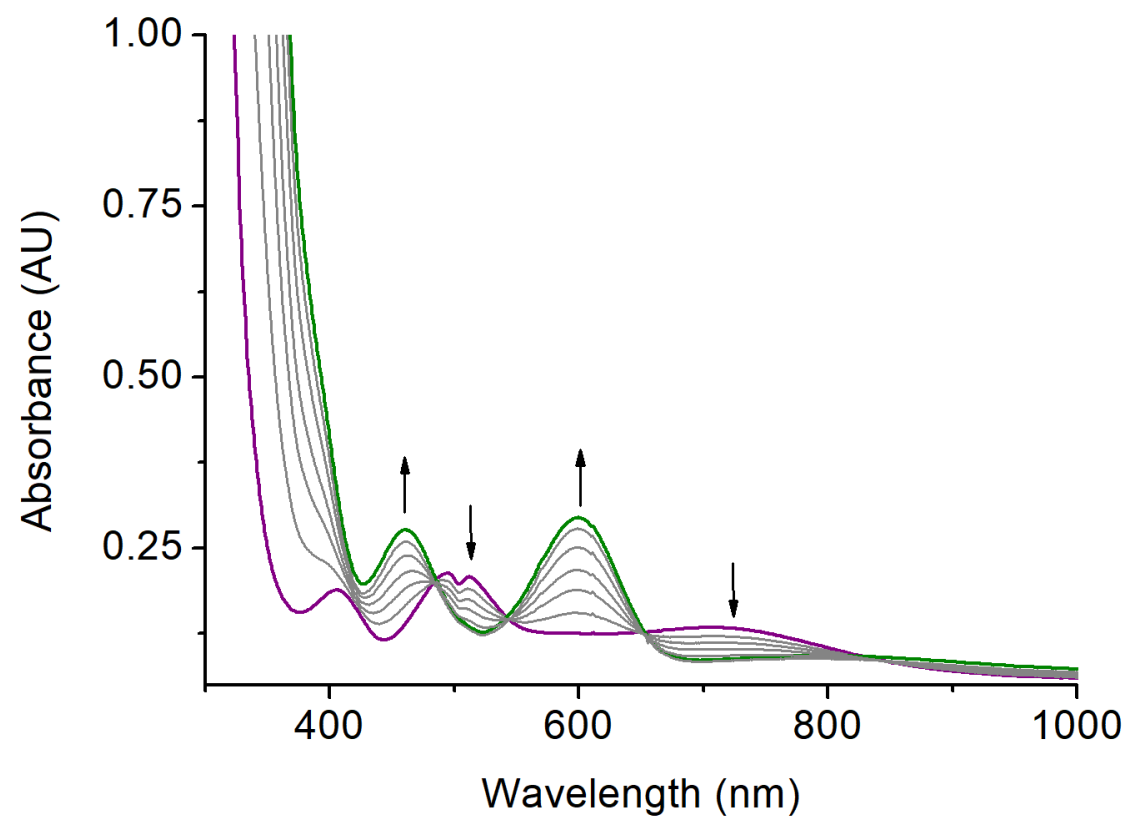

(b)

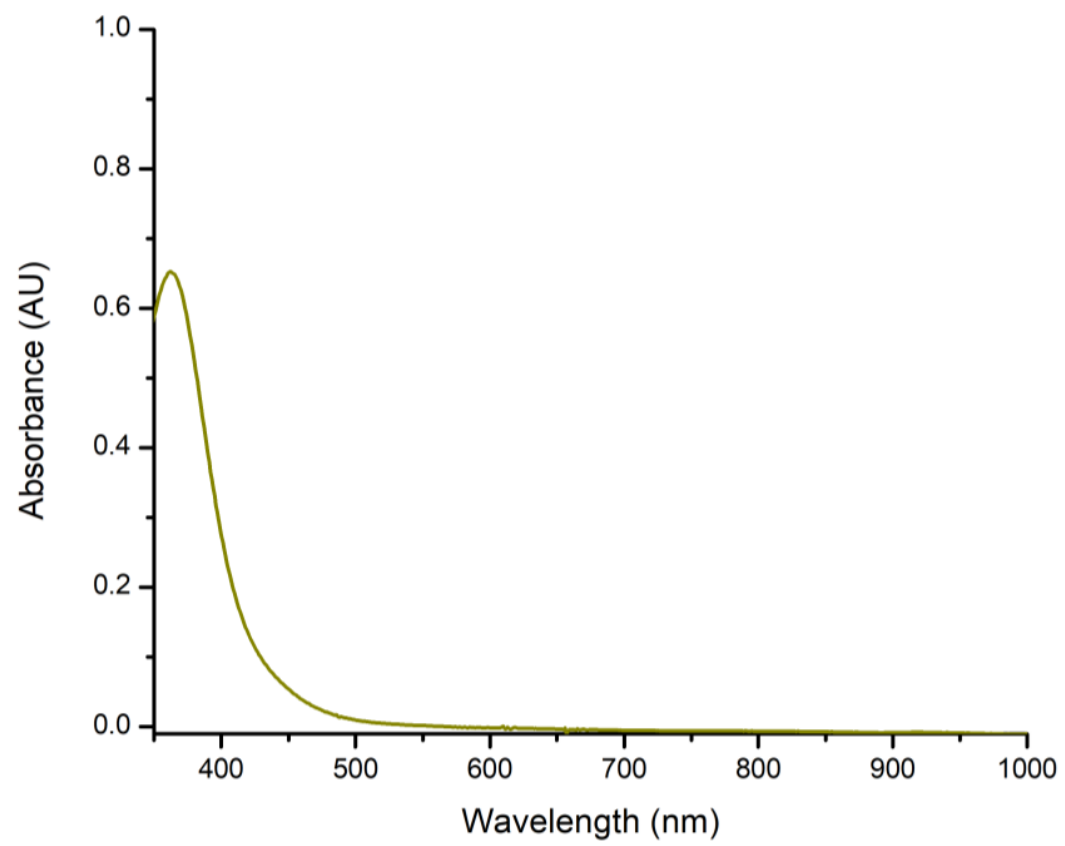

Figure S12. (a) Reaction of $\mathbf{5}^{\prime}$ with one equiv of HOTf in THF forming $\left[\mathrm{Mn}\left(\mathrm{BDP}^{\mathrm{Br}} \mathrm{P}\right)\left(\mathrm{H}_{2} \mathrm{O}\right)\right] \mathrm{OTf}\left(\mathbf{6}^{\prime}\right)$, which was subsequently warmed up. (b) $100 \mu \mathrm{L}$ of the resulting solution was added into an acetone solution of excess $\mathrm{NaI}$ to examine the yield of the produced $\mathrm{H}_{2} \mathrm{O}_{2}$. The theoretical concentration of the in situ generated $\mathrm{I}_{3}{ }^{-}$solution is $3.2 \times 10^{-5} \mathrm{M}$. The amount of the formed $\mathrm{I}_{3}{ }^{-}$was then quantified using its visible spectrum $\left(\lambda_{\max }=361 \mathrm{~nm}, \varepsilon=2.5\right.$ $\left.\times 10^{4} \mathrm{M}^{-1} \mathrm{~cm}^{-1}\right)$. 


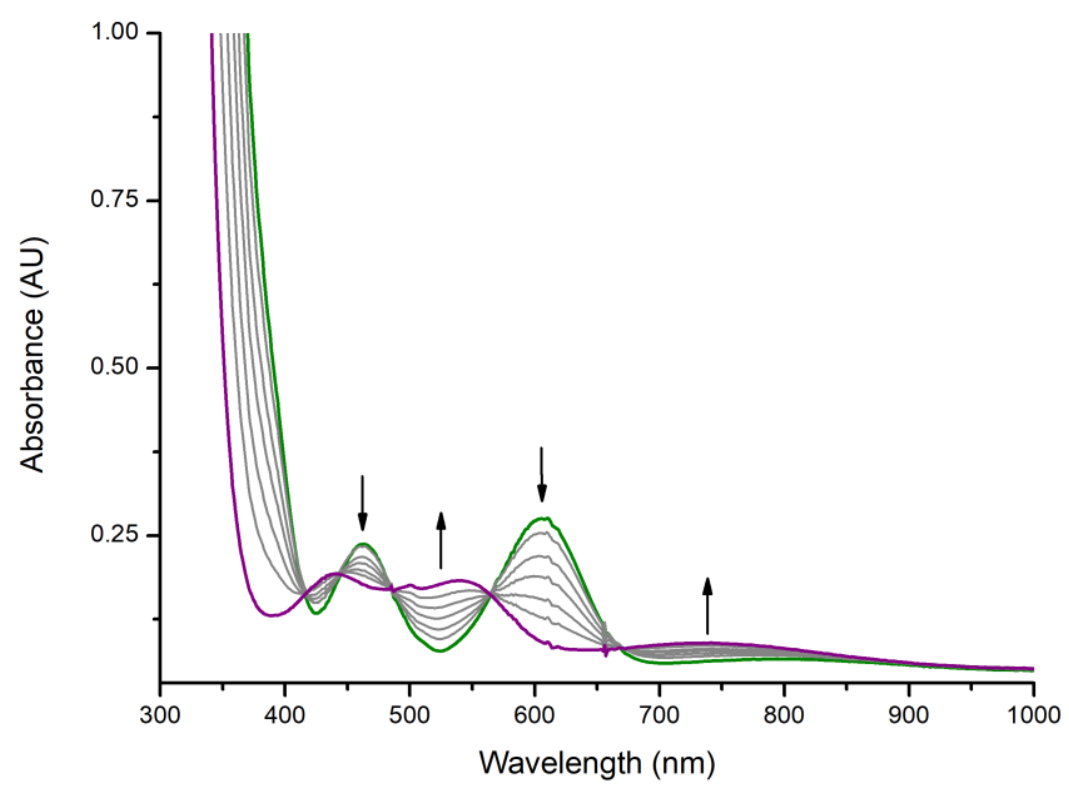

Figure S13. UV-vis spectra change monitored from the reaction of $\left[\mathrm{Mn}(\mathrm{BDPP})\left(\mathrm{H}_{2} \mathrm{O}\right)\right] \mathrm{OTf}(\mathbf{6})$ (green line, $1 \mathrm{mM}$ ) with excess $\mathrm{H}_{2} \mathrm{O}_{2} / \mathrm{NEt}_{3} 2: 1$ mixture in $\mathrm{THF}$ at $-90{ }^{\circ} \mathrm{C}$. The purple line represents $\mathrm{Mn}(\mathrm{BDPP})(\mathrm{OOH})(\mathbf{5})$. 

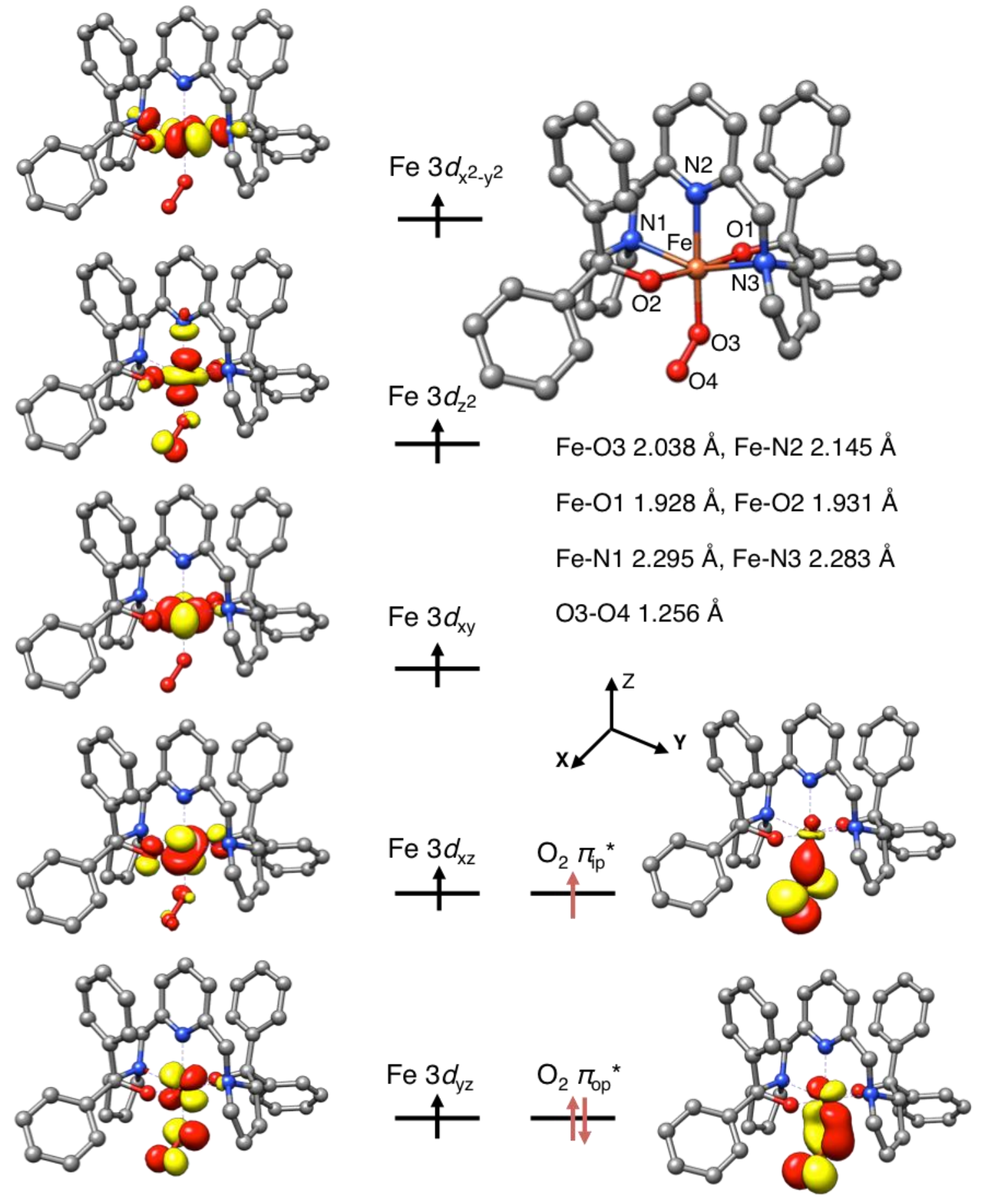

Figure S14. Computed structure of $S=3 \mathrm{Fe}^{\mathrm{III}}$-superoxo 3 with key geometric parameters and its schematic molecular orbital diagram. 

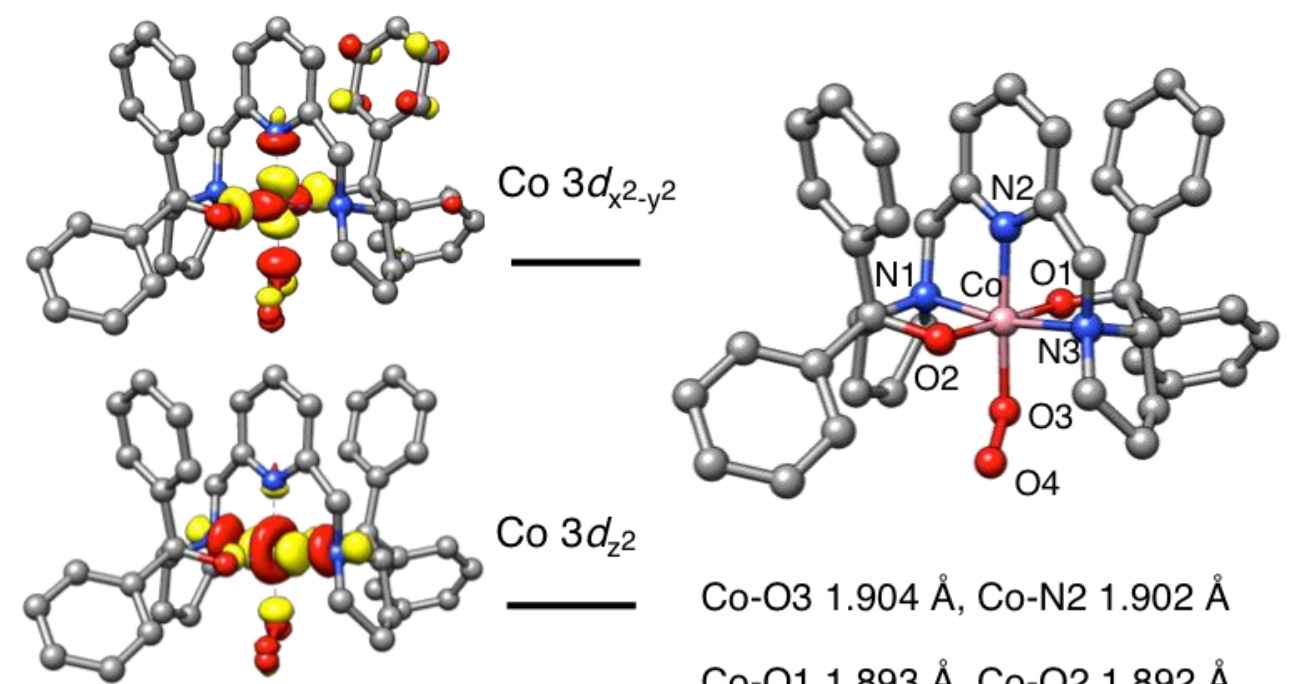

Co-O3 $1.904 \AA$ Å, Co-N2 $1.902 \AA$

Co-O1 1.893 Å, Co-O2 $1.892 \AA$

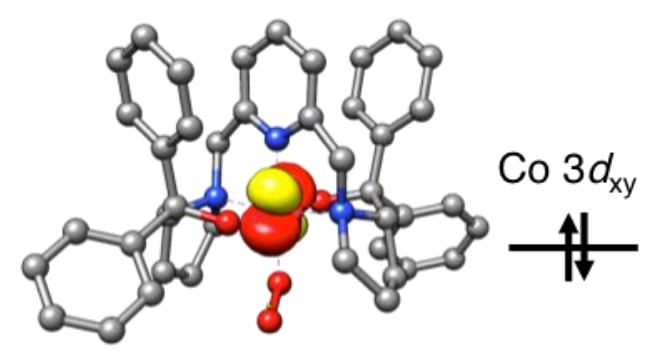

Co-N1 $2.015 \AA$, Co-N3 $2.012 \AA$

O3-O4 $1.298 \AA$
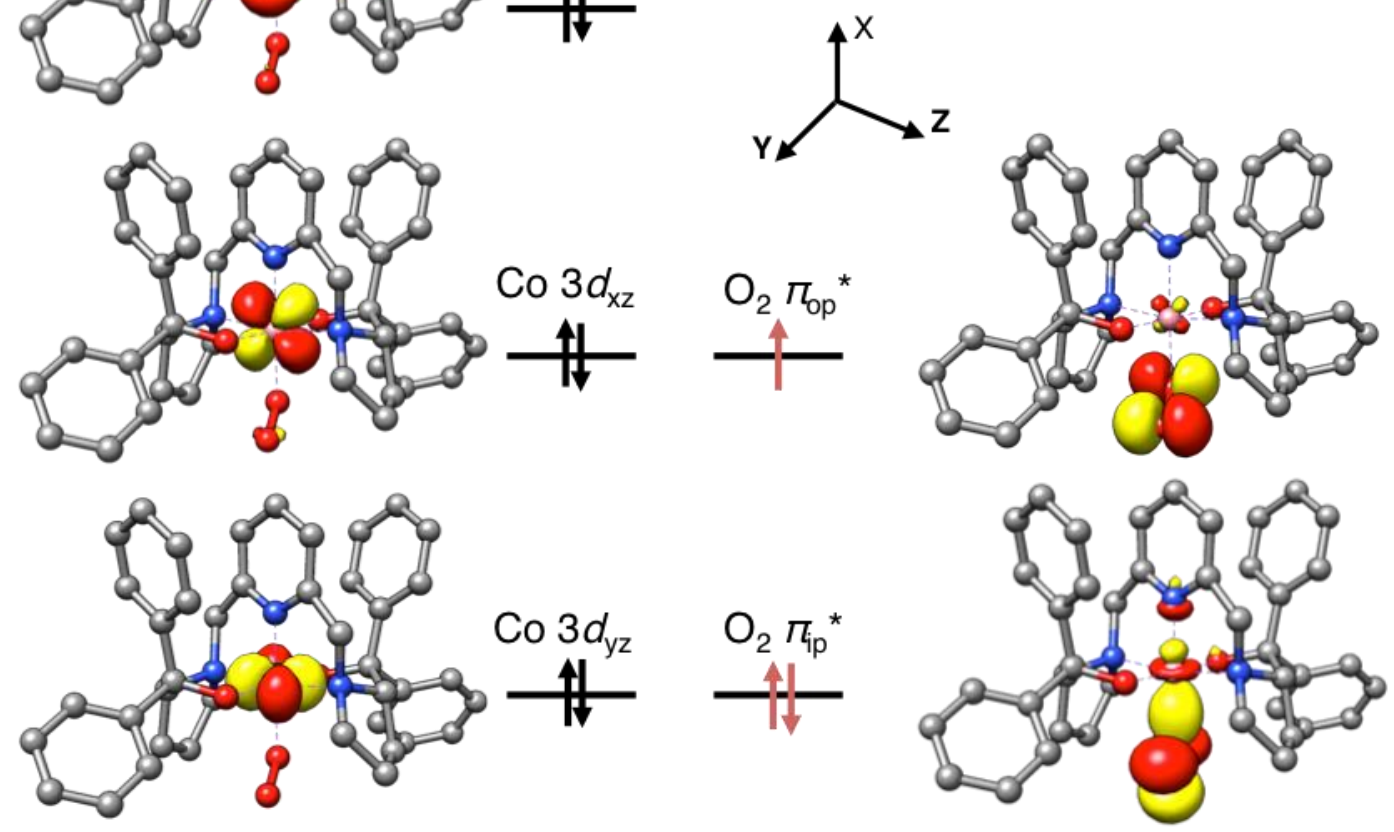

Figure S15. Computed structure of $S=1 / 2 \mathrm{Co}^{\mathrm{III}}$-superoxo 4 with key geometric parameters and its schematic molecular orbital diagram. 
For most transition metal complexes, the zero-field splitting arises from $2^{\text {nd }}$ order spin-oribit coupling between the ground and excited states. A negative $D$ value is usually found for a high spin $\mathrm{d}^{4}$ center in a elongated octahderal coordination environment, because

$$
D=\frac{1}{4} \frac{2}{\left({ }^{5} B_{2 g}\right)}+\frac{1}{16} \frac{2}{\left({ }^{5} E_{g}\right)} \frac{1}{4} \frac{2}{\left({ }^{3} E_{g}\right)}
$$

here $\zeta$ is the effective spin-orbit coupling constant of the metal center, $\Delta$ is the excitation energy of a given excited state.

The hyperfine coupling constant (HFC) is predicted to display a pattern of $\left|A_{x, y}\right|>\left|A_{z}\right|$, because

$$
\begin{aligned}
& A_{z}=A^{F C}+\frac{4}{7} P \\
& A_{x, y}=A^{F C} \frac{2}{7} P
\end{aligned}
$$

here $\mathrm{A}^{\mathrm{FC}}$ is the Fermi-contact contribution to the HFC and is always negative for mononuclear transition metal complexes, $\kappa<1$ is the orbital reduction factor and $\mathrm{P}$ is the proportional constant of the nucleus and is negative for ${ }^{55} \mathrm{Mn}$. Because the intrinsic $\mathrm{g}$ values of $\mathbf{2}$ and $\mathbf{2}^{\prime}$ are very close to 2 , the orbital contribution to the HFC is negligible.
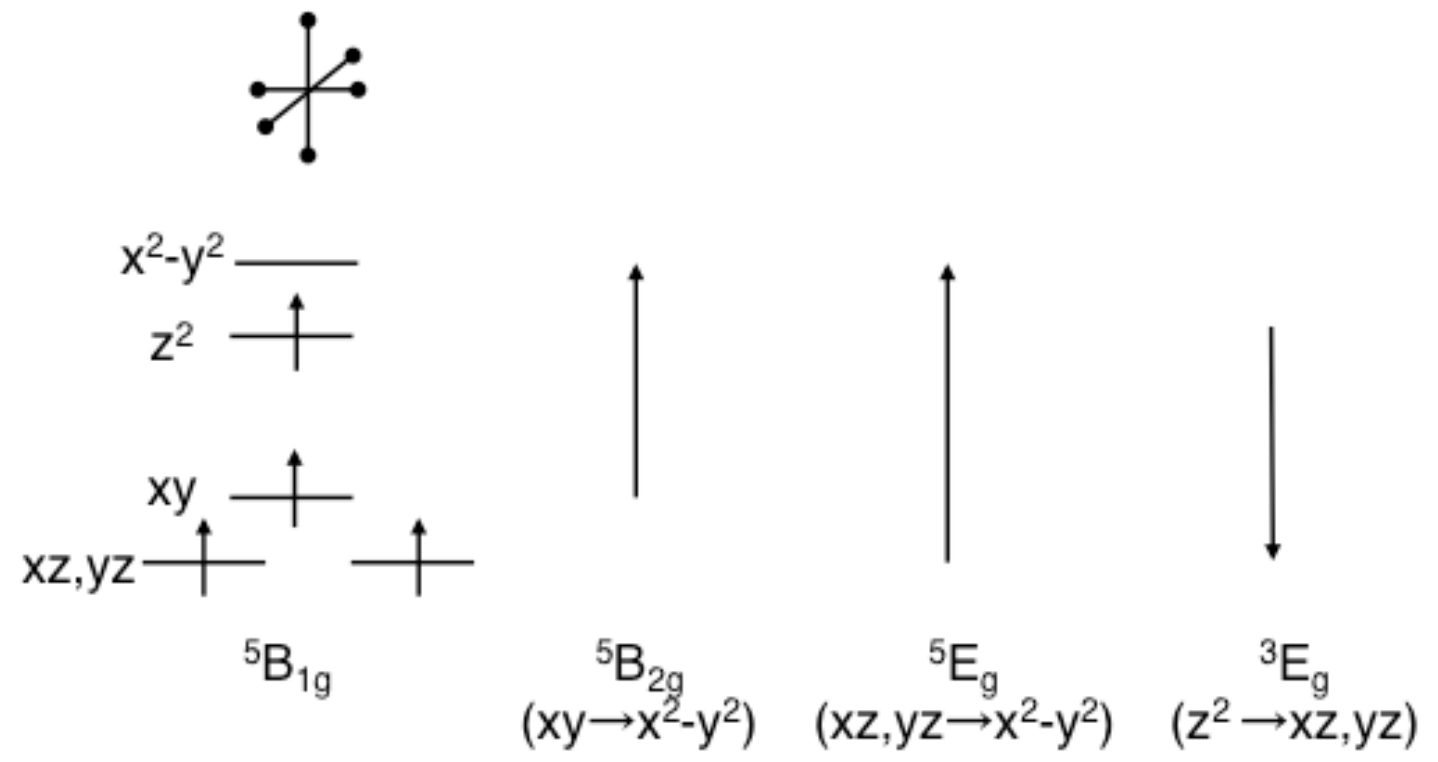

Figure S16. Correlation of the coordination geometry of $\mathbf{2}^{\prime}$ with its spin Hamiltonian parameters. The ground state of a high spin $\mathrm{d}^{4}$ center in a elongated octahderal coordination environment and low-lying excited states in $D_{4 \mathrm{~h}}$ symmetry. 
A kinetic titration with $\sim 2 \times 10^{-4} \mathrm{M}$ of $2^{\prime}$ and TEMPOH at 1.0, 1.5, 2.0, 2.5 equivalent concentrations was performed. All these numbers are based on a $100 \%$ yield in their preparation processes. The concentration of $\mathbf{2}^{\prime}$ was corrected to $1.86 \times 10^{-4} \mathrm{M}$ using the EPR data ( $93 \%$ yield, Figure S10), while the concentrations of TEMPO-H remained unknown. We then performed a titration with the nominal 1.0, 1.5, 2.0, 2.5 equiv of TEMPO-H and fitted to the kinetic model to obtain the actual value of $n$ and rate constant, $k$. The actual value of $n$ against the nominal $n$ was plotted and we found that the yield of TEMPO-H generation was $\sim 80 \%$, which is consistent with the value extracted from the ${ }^{1} \mathrm{H}$ NMR spectrum (Figure S18). Therefore, the actual amount of the employed TEMPO-H is $0.8,1.2,1.6$, and 2.0 equivalents, respectively.

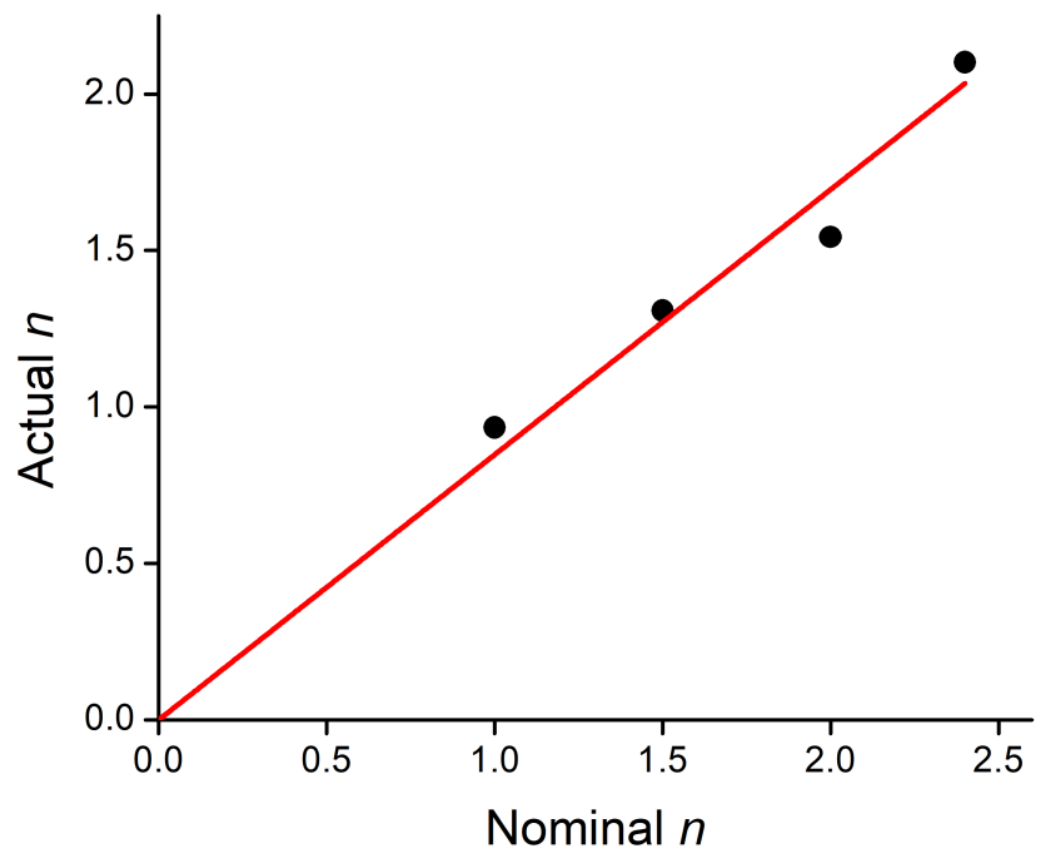

Figure S17. The linear plot of the actual value of $n$ against the nominal $n$. 

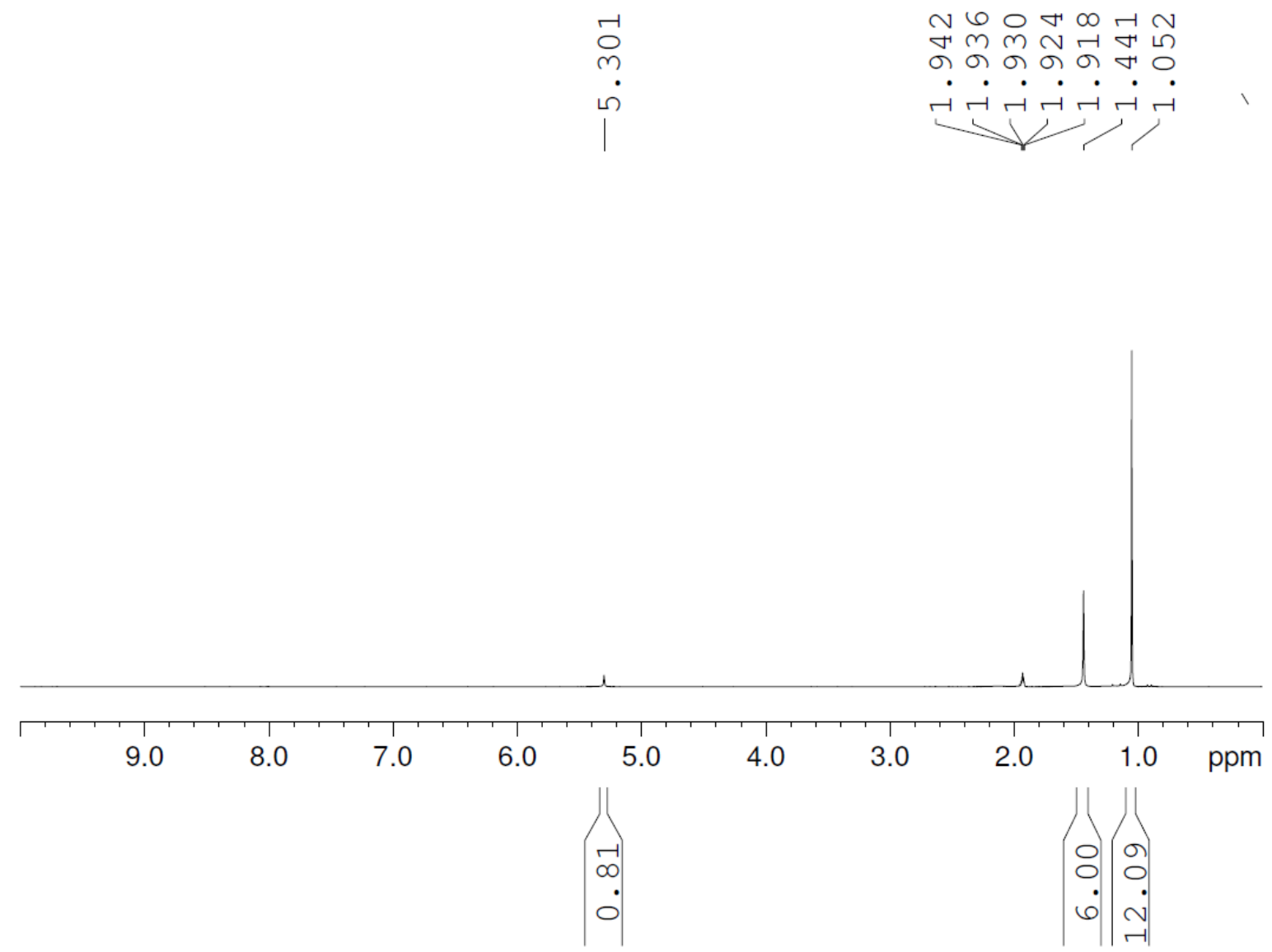

Figure S18. ${ }^{1} \mathrm{H}$ NMR spectrum of the prepared TEMPO-H in $\mathrm{CD}_{3} \mathrm{CN}$. 
Table S1. Mn-O bond distances of Mn- $\left(\mathrm{O}_{2}\right)$, Mn-OOR, and Mn-OR ( $=\mathrm{H}, \mathrm{Me}, \mathrm{Ph}, \mathrm{Ph}-$ ${ }^{p} \mathrm{NO}_{2}$ ) complexes.

\begin{tabular}{|c|c|c|}
\hline Complex & $\mathrm{Mn}-\mathrm{O}(\AA)$ & ref. \\
\hline$\left[\mathrm{Mn}\left(\mathrm{O}_{2}\right)(\mathrm{TPP})\right]^{-}$ & $1.888 / 1.901$ & 2 \\
\hline \multirow[t]{2}{*}[\mathrm{Mn}(\mathrm{O}_{2})(\mathrm{Tp}^{\mathrm{iPr}2})(\mathrm{pz}^{\mathrm{iPr}2})]{} & $1.850 / 1.851($ brown $)$ & 3 \\
\hline & 1.841/1.878(blue) & \\
\hline $\mathrm{Tp}{ }^{\mathrm{iPr} 2} \mathrm{Mn}\left(\eta^{2}-\mathrm{O}_{2}\right)\left(\mathrm{im}^{\mathrm{Me}} \mathrm{H}\right)$ & $1.872 / 1.838$ & 4 \\
\hline$\left[\mathrm{Mn}\left(\mathrm{O}_{2}\right)(\mathrm{TMC})\right]^{+}$ & 1.884 & 5 \\
\hline$\left[\mathrm{Mn}\left(\mathrm{O}_{2}\right)(13-\mathrm{TMC})\right]^{+}$ & $1.855 / 1.863$ & 6 \\
\hline$\left[\mathrm{Mn}\left(\mathrm{O}_{2}\right)(12-\mathrm{TMC})\right]^{+}$ & 1.853 & 7 \\
\hline$\left[\mathrm{Mn}^{\mathrm{III}}\left(\mathrm{OO}^{t} \mathrm{Bu}\right)\left(\mathrm{S}^{\mathrm{Me} 2} \mathrm{~N}_{4}(\text { QuinoEN })\right)\right]^{+}$ & 1.861 & 8 \\
\hline$\left[\mathrm{Mn}^{\mathrm{III}}(\mathrm{OOCm})\left(\mathrm{S}^{\mathrm{Me} 2} \mathrm{~N}_{4}(\text { QuinoEN })\right)\right]^{+}$ & 1.841 & 8 \\
\hline$\left[\mathrm{Mn}^{\mathrm{III}}\left(\mathrm{OO}^{t} \mathrm{Bu}\right)\left(\mathrm{S}^{\mathrm{Me} 2} \mathrm{~N}_{4}(\text { QuinoPN })\right)\right]^{+}$ & 1.840 & 9 \\
\hline$\left[\mathrm{Mn}^{\mathrm{III}}\left(\mathrm{OO}^{\mathrm{t}} \mathrm{Bu}\right)\left(\mathrm{S}^{\mathrm{Me} 2} \mathrm{~N}_{4}(6-\mathrm{Me}-\mathrm{DPEN})\right)\right]^{+}$ & 1.853 & 9 \\
\hline$\left[\mathrm{Mn}^{\mathrm{III}}(\mathrm{OOCm})\left(\mathrm{S}^{\mathrm{Me} 2} \mathrm{~N}_{4}(6-\mathrm{Me}-\mathrm{DPEN})\right)\right]^{+}$ & 1.848 & 9 \\
\hline$\left[\mathrm{Mn}^{\mathrm{III}}\left(\mathrm{OO}^{t} \mathrm{Bu}\right)\left(\mathrm{S}^{\mathrm{Me} 2} \mathrm{~N}_{4}(6-\mathrm{Me}-\mathrm{DPPN})\right)\right]^{+}$ & 1.843 & 9 \\
\hline$\left[\mathrm{Mn}_{2}{ }^{\mathrm{III}}(\text { trans-1,2-O } 2)\left(\mathrm{S}^{\mathrm{Me} 2} \mathrm{~N}_{4}(6-\mathrm{Me}-\mathrm{DPEN})\right)_{2}\right]^{2+}$ & 1.832 & 10 \\
\hline$\left[\mathrm{Mn}^{\mathrm{III}}\left(\mathrm{S}^{\mathrm{Me} 2} \mathrm{~N}_{4}(\operatorname{tren})\left(\mathrm{OPh}_{-}{ }^{p} \mathrm{NO}_{2}\right)\right)\right]^{+}$ & 1.901 & 11 \\
\hline$\left[\mathrm{Mn}^{\mathrm{III}}\left(\mathrm{S}^{\mathrm{Me} 2} \mathrm{~N}_{4}(\operatorname{tren})(\mathrm{OPh})\right)\right]^{+}$ & 1.867 & 11 \\
\hline$\left[\mathrm{Mn}^{\mathrm{III}}\left(\mathrm{S}_{\mathrm{Me} 2} \mathrm{~N}_{4}(\operatorname{tren})(\mathrm{OMe})\right)\right]^{+}$ & 1.836 & 11 \\
\hline$\left[\mathrm{Mn}^{\mathrm{III}}\left(\mathrm{S}_{\mathrm{Me} 2} \mathrm{~N}_{4}(\operatorname{tren})\right)(\mathrm{OH})\right]^{+}$ & 1.854 & 11 \\
\hline
\end{tabular}


Table S2. The O-O bond length of metal-superoxo complexes.

\begin{tabular}{llc}
\hline complex & $\mathrm{O}-\mathrm{O}(\AA)$ & ref. \\
\hline$\left[\mathrm{Cu}^{\mathrm{II}}\left(\mathrm{TMG}_{3} \text { tren }\right)\left(\eta^{1}-\mathrm{O}_{2}{ }^{\circ}\right)\right]^{+}$ & $1.280(3)$ & 12 \\
{$\left[\mathrm{Cr}{ }^{\mathrm{III}}(14-\mathrm{TMC})\left(\mathrm{O}_{2}\right)(\mathrm{Cl})\right]^{+}$} & $1.231(6)$ & 13 \\
{$\left[\mathrm{Fe}^{\mathrm{III}}(\mathrm{TAML})\left(\mathrm{O}_{2}\right)\right]^{2-}$} & $1.323(3) / 1.306(7)$ & 14 \\
$\mathrm{Co}^{\mathrm{III}}\left(\mathrm{Tp}^{\mathrm{Me} 2}\right)\left(\mathrm{L}^{\mathrm{Ph}}\right)\left(\mathrm{O}_{2}{ }^{*}\right)$ & $1.301(5)$ & 15 \\
\hline
\end{tabular}

Table S3. Computed Spectroscopic Parameters for $\mathbf{2}^{\prime}$ and $\mathbf{5}^{\prime}$.

\begin{tabular}{lll}
\hline & $\mathbf{2}^{\prime}$ & $\mathbf{5}^{\prime}$ \\
\hline$D\left(\mathrm{~cm}^{-1}\right), E / D$ & $-3.1,0.08$ & $-2.8,0.11$ \\
$A_{\mathrm{x}, \mathrm{y}, \mathrm{z}}(\mathrm{MHz})$ & $-194,-183,-60$ & $-166,-164,-47$ \\
\hline
\end{tabular}

Table S4. Metal-superoxo bond lengths of mononuclear metal superoxo complexes by the B3LYP calculations

\begin{tabular}{ll}
\hline Complex & Metal-O $(\AA)$ \\
\hline $\mathrm{Mn}^{\mathrm{III}}(\mathrm{BDPP})\left(\mathrm{O}_{2}{ }^{\circ}\right)(\mathbf{2}) S=3 / 2$ & 1.932 \\
$\mathrm{Mn}^{\mathrm{III}}\left(\mathrm{BDP}^{\mathrm{Br}} \mathrm{P}\right)\left(\mathrm{O}_{2}{ }^{\circ}\right)\left(\mathbf{2}^{\prime}\right) S=3 / 2$ & 1.937 \\
$\mathrm{Fe}^{\mathrm{III}}(\mathrm{BDPP})\left(\mathrm{O}_{2}{ }^{\circ}\right)(\mathbf{3}) S=3$ & 2.038 \\
$\mathrm{Fe}^{\mathrm{III}}(\mathrm{BDPP})\left(\mathrm{O}_{2}{ }^{\circ}\right) S=2$ & 1.927 \\
$\mathrm{Co}^{\mathrm{III}}(\mathrm{BDPP})\left(\mathrm{O}_{2}{ }^{\circ}\right)(\mathbf{4}) S=1 / 2$ & 1.904 \\
\hline
\end{tabular}

For 2, $\mathbf{2}^{\prime}$ and 4 , the strongly antibonding $\sigma^{*}$ orbital with respect to the metal-superoxo interaction is vacant (Fig. 2, S15 and S16), but it is singly occupied for 3 (S15). In fact, the computed Fe-superoxo bond distance for the quintet state of $\mathbf{3}$, which contains a high spin ferric center antiferromagnetic coupled to a superoxo radical, is comparable to those estimated for $\mathbf{2}$, $\mathbf{2}^{\prime}$ and 4. This observation reflects the tradeoff between the enhanced Lewis acidity of $\mathrm{Fe}^{\mathrm{III}}$ relative to $\mathrm{Mn}^{\mathrm{III}}$ and the different occupation of the $\sigma^{*}$ orbital. Thus, the weaker metal-superoxo bonding in $\mathbf{3}$ arises from the synergistic effect of its high spin center and the ferromagnetic coupling between the metal center and the superoxo ligand. ${ }^{16}$ 
Table S5. Coordinates of the optimized structures:

$\mathrm{Mn}^{\mathrm{III}}(\mathrm{BDPP})\left(\mathrm{O}_{2}{ }^{\circ}\right)(\mathbf{2})$ :

\begin{tabular}{|c|c|c|c|}
\hline Mn & -1.49310707853518 & 10.62070401636536 & 599976055327 \\
\hline $\mathrm{H}$ & -0.84052727955616 & 17.89033641991843 & 10.90627631645225 \\
\hline $\mathrm{H}$ & 5.01857969650649 & 11.26080589118739 & 10.73402742227351 \\
\hline $\mathrm{H}$ & 2.07503813535693 & 4.69710544057160 & 7.17726564893587 \\
\hline $\mathrm{H}$ & -6.52629979160194 & 5.68105357315492 & 5.16186779040529 \\
\hline $\mathrm{O}$ & -3.24166978381150 & 11.38799509030409 & 8.02900524329407 \\
\hline $\mathrm{O}$ & -3.67414027267409 & 12.38540561576013 & 8.73889422220261 \\
\hline$C$ & 0.14622534588044 & 12.91909531175147 & 8.89420317066122 \\
\hline $\mathrm{C}$ & -0.10175819939280 & 14.32777139554240 & 9.48067608502189 \\
\hline $\mathrm{C}$ & -1.25039677166967 & 14.56342102057798 & 10.24525422320290 \\
\hline $\mathrm{H}$ & -1.93956318762866 & 13.73466405363135 & 10.40731447700653 \\
\hline $\mathrm{C}$ & -1.51079762708346 & 15.83900015954261 & 10.75611094238972 \\
\hline $\mathrm{H}$ & -2.41295124351058 & 16.01080940148165 & 11.34932715597300 \\
\hline $\mathrm{C}$ & -0.63111416155302 & 78040468 & 10.50572 \\
\hline $\mathrm{C}$ & 0.51685892349555 & 16.66620033960102 & 9.74001798282374 \\
\hline $\mathrm{H}$ & 1.21463140476065 & 17.48265789959905 & 9.53463229871925 \\
\hline $\mathrm{C}$ & 0.78122712437718 & 15.38973374736062 & 9.23787936590845 \\
\hline $\mathrm{H}$ & 1.68990679752214 & 15.22651825550652 & 8.65561492712120 \\
\hline $\mathrm{C}$ & 1.54887194262492 & 12.46969719222107 & 9.36596705764002 \\
\hline $\mathrm{C}$ & 2.73958683059272 & 12.79531667872422 & 8.70057964326542 \\
\hline $\mathrm{H}$ & 2.71500331928337 & 13.37736517287429 & 7.77844432724151 \\
\hline $\mathrm{C}$ & 3.97994414641610 & 12.36899942252690 & 9.18659308766635 \\
\hline $\mathrm{H}$ & 4.88957689851170 & 12.63625731700067 & 8.64209549326547 \\
\hline $\mathrm{C}$ & 4.05404321691218 & 11.60804093254889 & 10.35443668331921 \\
\hline $\mathrm{C}$ & 2.87486989710584 & 11.29685339035780 & 11.04027114237799 \\
\hline $\mathrm{H}$ & 2.92024551233145 & 10.70946489236244 & 11.96124903998786 \\
\hline $\mathrm{C}$ & 1.64258364048388 & 11.73112271640251 & 10.55379545548812 \\
\hline $\mathrm{H}$ & 0.71758323045842 & 11.49274445394469 & 11.07828216750919 \\
\hline $\mathrm{C}$ & 0.03054424875374 & 13.01350794885808 & 7.33871011130346 \\
\hline $\mathrm{H}$ & 0.92560174913585 & 13.49914005568553 & 6.91415736805470 \\
\hline$C$ & -1.22935184923215 & 13.78141861355586 & 6.88475407665857 \\
\hline & -1.97189364236985 & 13.79093638171272 & 7.69038637713665 \\
\hline
\end{tabular}




\begin{tabular}{|c|c|c|c|}
\hline $\mathrm{H}$ & -0.97223894775023 & 14.82627823552659 & 6.66723557592898 \\
\hline C & -1.77725422560312 & 13.02753657281822 & 5.65407694949191 \\
\hline & -2.75641374083611 & 12.58670119431629 & 5.88264408708293 \\
\hline & -1.89349957099467 & 13.67868948992305 & 4.77560516984310 \\
\hline 4 & -0.75659811348951 & 11.92502328627028 & 5.39325748774536 \\
\hline ப & 0.01636172848060 & 12.26694458237399 & 4.67525449574295 \\
\hline $\mathrm{H}$ & -1.19829923760710 & 10.99144994844540 & 5.01360615265501 \\
\hline $\mathrm{C}$ & 1.06911767983923 & 10.84907647110080 & 6.56677054854184 \\
\hline $\mathrm{H}$ & 1.95803285176790 & 11.45646027571068 & 6.31653191108363 \\
\hline $\mathrm{H}$ & 0.89929909586749 & 10.17687255315494 & 5.70992296512588 \\
\hline $\mathrm{C}$ & 1.37718178772157 & 9.94743723234859 & 7.73670328778378 \\
\hline $\mathrm{C}$ & 2.63036273687908 & 9.35639310753699 & 7.89098221968994 \\
\hline $\mathrm{H}$ & 3.44894682225362 & 9.63872870375137 & 7.22748523312434 \\
\hline C & 2.80255281499032 & 8.40870985846851 & 8.89768697164028 \\
\hline $\mathrm{H}$ & 3.76845122084391 & 7.91949326496361 & 9.03974231178131 \\
\hline $\mathrm{C}$ & 1.73067134844477 & 8.09084462052730 & 9.72844525722570 \\
\hline $\mathrm{H}$ & 1.82770650367504 & 7.34300276009553 & 10.51720185330483 \\
\hline $\mathrm{C}$ & 0.52183339522911 & 8.76020374383102 & 9.54559777832321 \\
\hline $\mathrm{C}$ & -0.62042152643410 & 8.60130967639636 & 10.51590417775830 \\
\hline $\mathrm{H}$ & -0.66663788248012 & 7.55661159889587 & 10.87325597294391 \\
\hline $\mathrm{H}$ & -0.35694430627211 & 9.21816904305559 & 11.39034391872744 \\
\hline $\mathrm{C}$ & -2.74101448079485 & 9.55216783923231 & 11.17267865456405 \\
\hline $\mathrm{H}$ & -2.35186068527505 & 10.52434641557261 & 11.50887011882127 \\
\hline $\mathrm{H}$ & -2.65827114126878 & 8.83461371509353 & 12.01292500613640 \\
\hline $\mathrm{C}$ & -4.17621664612315 & 9.60827754301264 & 10.64886547286976 \\
\hline $\mathrm{H}$ & -4.44157906791470 & 10.63166304101441 & 10.35420249288793 \\
\hline $\mathrm{H}$ & -4.88441878685141 & 9.28416969140637 & 11.42500795408573 \\
\hline $\mathrm{C}$ & -4.18950268671887 & 8.67571007509249 & 9.42026330828641 \\
\hline $\mathrm{H}$ & -4.43180365654426 & 9.24320804804754 & 8.51485526213079 \\
\hline $\mathrm{H}$ & -4.92545405682129 & 7.86561554583102 & 9.50545123073820 \\
\hline $\mathrm{C}$ & -2.77069760270976 & 8.08653454225467 & 9.30624534264463 \\
\hline $\mathrm{H}$ & -2.73621451277729 & 7.12167837903978 & 9.83941099533761 \\
\hline $\mathrm{C}$ & -2.31040895284103 & 7.90001149803476 & 7.82128606759063 \\
\hline $\mathrm{C}$ & -1.07750853439159 & 6.97291040085040 & 7.68970790982256 \\
\hline$C$ & -0.17128687132606 & 7.21511137761188 & 6.64631592956467 \\
\hline
\end{tabular}




\begin{tabular}{|c|c|c|c|}
\hline $\mathrm{H}$ & -0.36474146799768 & 8.06470797694014 & 5.99178428969691 \\
\hline $\mathrm{C}$ & 0.95143468263482 & 6.40845123817842 & 6.46143894261341 \\
\hline $\mathrm{H}$ & 1.64565259605728 & 6.62661718534472 & 5.64537207714192 \\
\hline $\mathrm{C}$ & 1.19475073916931 & 5.32914432652405 & 7.31853980673017 \\
\hline $\mathrm{C}$ & 0.29061294423970 & 5.06106776063986 & 8.34797061782119 \\
\hline $\mathrm{H}$ & 0.45851731829559 & 4.21467797376120 & 9.01920607693330 \\
\hline $\mathrm{C}$ & -0.83338430907712 & 5.87509834869954 & 8.52874505418955 \\
\hline $\mathrm{H}$ & -1.52070652810445 & 5.64004213160069 & 9.34287847250619 \\
\hline $\mathrm{C}$ & -3.47360557149796 & 7.23740549304771 & 7.04698724005872 \\
\hline $\mathrm{C}$ & -3.94687175692993 & 5.96061622361439 & 7.38178550972141 \\
\hline $\mathrm{H}$ & -3.46126792055097 & 5.38588801440165 & 8.17257289458921 \\
\hline $\mathrm{C}$ & -5.04258777707580 & 5.40654561529835 & 6.71644512752867 \\
\hline $\mathrm{H}$ & -5.40984463131399 & 4.41920776592237 & 7.00850828330096 \\
\hline $\mathrm{C}$ & -5.67193196848414 & 6.11499857852955 & 5.68738552998992 \\
\hline $\mathrm{C}$ & -5.19788026001982 & 7.38186899170038 & 5.33982568349371 \\
\hline $\mathrm{H}$ & -5.68621831426943 & 7.94838012985158 & 4.54232610685194 \\
\hline $\mathrm{C}$ & -4.11182467742997 & 7.94251536066904 & 6.02026925654814 \\
\hline $\mathrm{H}$ & -3.74931301861282 & 8.94133408108303 & 5.77955364122230 \\
\hline $\mathrm{N}$ & -0.13031332963904 & 11.67191750508400 & 6.70888526860159 \\
\hline $\mathrm{N}$ & 0.37594260786863 & 9.65378823960454 & 8.56720800832749 \\
\hline $\mathrm{N}$ & -1.92108157411194 & 9.06864407721198 & 10.03754111049954 \\
\hline $\mathrm{O}$ & -0.83880228711981 & 12.04660752668565 & 9.35677533812856 \\
\hline $\mathrm{O}$ & -2.04512742008841 & 9.15377754296478 & 7.27301730742646 \\
\hline \multicolumn{4}{|c|}{$\mathrm{Mn}^{\mathrm{III}}\left(\mathrm{BDP}^{\mathrm{Br}} \mathrm{P}\right)\left(\mathrm{O}_{2}{ }^{\circ}\right)\left(\mathbf{2}^{\prime}\right):$} \\
\hline $\mathrm{Mn}$ & -1.51895213447833 & 10.63764487741676 & 8.38349320521501 \\
\hline $\mathrm{Br}$ & -0.69886924630518 & 18.67328640191320 & 11.34082736319431 \\
\hline $\mathrm{Br}$ & 5.83028820189951 & 11.36773456966083 & 11.04635755294360 \\
\hline $\mathrm{Br}$ & 2.56450232421277 & 4.10185962226560 & 6.86817348804085 \\
\hline $\mathrm{Br}$ & -7.04650463447380 & 5.32476516131998 & 4.52275941149838 \\
\hline $\mathrm{O}$ & -3.25046321243856 & 11.46928666281454 & 8.13180343235474 \\
\hline $\mathrm{O}$ & -3.70563578810636 & 12.23377387734376 & 9.07203661888293 \\
\hline $\mathrm{C}$ & 0.16403311485209 & 12.98555612225928 & 8.84722230288068 \\
\hline $\mathrm{C}$ & -0.07967251569240 & 14.41405533912043 & 9.4089358477097 \\
\hline
\end{tabular}




\begin{tabular}{|c|c|c|c|}
\hline $\mathrm{C}$ & -1.14945432086786 & 14.65182633004714 & 56437410 \\
\hline & -1.82859676885991 & 13.82872577998237 & 10.50831593980150 \\
\hline & -1.34886562116418 & 15.91552360797323 & 10.84999674238523 \\
\hline & -2.18025612174042 & 16.08700596102225 & 11.53671489706009 \\
\hline & -0.47157408926745 & 16.95524776419527 & 10.53618233400430 \\
\hline & 0.58395009786854 & 16.75192216605784 & 9.64282181859021 \\
\hline & 1.25928086704623 & 17.57127996901351 & 9.38945836919177 \\
\hline & 0.77557710807385 & 15.48289661721714 & 9.09433367005859 \\
\hline & 1.62491870271034 & 15.33539653698615 & 8.42407692688164 \\
\hline & 1.58896229011548 & 12.59263542189372 & 9.33945946164418 \\
\hline & 2.77233638691858 & 12.90972316384025 & 8.65343135039126 \\
\hline & 2.73877140470569 & 13.43117095736294 & 7.69547177903210 \\
\hline & 4.03164490604418 & 12.56340866797068 & 9.15513013643115 \\
\hline & 4.93424262047361 & 12.81643446378240 & 8.59591219812835 \\
\hline & 4.12056971861702 & 11.89000717167201 & 10.37276009886100 \\
\hline & 2.96332172004586 & 11.59097211465942 & 11.09806655048970 \\
\hline & 3.03874811976159 & 11.07961871509721 & 12.05948095232366 \\
\hline & 1.71680208039033 & 11.94723493165507 & 10.57986661930432 \\
\hline & 0.80930801088285 & 11.71676691565925 & 11.13995191228821 \\
\hline & 0.03915535867764 & 13.01807686338296 & 7.28430485676101 \\
\hline & 0.93392518274367 & 13.48158333401761 & 6.83245170734228 \\
\hline & -1.21792363020898 & 13.77610768830851 & 6.7910691080549 \\
\hline 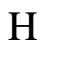 & -1.97154596558760 & 13.82366672656190 & 7.58641731724972 \\
\hline & -0.95650898762018 & 14.81123195254483 & 6.53254665537519 \\
\hline & -1.75507939939716 & 12.98006021293985 & 5.57936828791 \\
\hline & -2.73844916999840 & 12.54759768381982 & 5.810405923215 \\
\hline & -1.86396403763088 & 13.60179200678189 & 4.67842686101875 \\
\hline & -0.72959721525393 & 11.86969403675116 & 5.36723571121618 \\
\hline & 0.05678616732264 & 12.19402589379667 & 4.65513599591034 \\
\hline & -1.16526075428505 & 10.92927984698939 & 4.9967044938343 \\
\hline & 1.06456732236963 & 10.80229164895816 & 6.60948810095341 \\
\hline & 1.96510751655431 & 11.38854198747787 & 6.3480232265600 \\
\hline & 0.89775089738668 & 10.10765300890686 & 5.76953491118417 \\
\hline & 1.35690506111960 & 9.92536409352627 & 7.81073801149918 \\
\hline & 2.61400521721329 & 9.34588580703825 & 7.9950413292153 \\
\hline
\end{tabular}




\begin{tabular}{|c|c|c|c|}
\hline [ & 3.43755478309707 & 9.60684445798512 & 7.32763960927968 \\
\hline $\mathrm{C}$ & 2.78905786223767 & 8.43842633039606 & 9.03964476958529 \\
\hline $\mathrm{H}$ & 3.76115559140527 & 7.97116587299995 & 9.21234661463829 \\
\hline $\mathrm{C}$ & 1.70944611929002 & 8.13387575382380 & 9.86716390001944 \\
\hline $\mathrm{H}$ & 1.81094277550215 & 7.41776407882897 & 10.68539352730576 \\
\hline $\mathrm{C}$ & 0.48770589893565 & 8.77217970270913 & 9.64109699885035 \\
\hline $\mathrm{C}$ & -0.67704941577576 & 8.58453665479576 & 10.58838263871250 \\
\hline $\mathrm{H}$ & -0.70106651169894 & 7.53703303114839 & 10.94380607193627 \\
\hline $\mathrm{H}$ & -0.44322248354237 & 9.19857143792281 & 11.47409081902687 \\
\hline $\mathrm{C}$ & -2.82894904484766 & 9.43686618944339 & 11.26576831970328 \\
\hline $\mathrm{H}$ & -2.50068100016645 & 10.42788558044328 & 11.61231037992928 \\
\hline $\mathrm{H}$ & -2.67910689732079 & 8.71703005504197 & 12.09432528065135 \\
\hline $\mathrm{C}$ & -4.28317353294754 & 9.40310568190992 & 10.78605524801321 \\
\hline $\mathrm{H}$ & -4.66293109921776 & 10.41753699631108 & 10.60648219931047 \\
\hline $\mathrm{H}$ & -4.92761456753490 & 8.93523755851250 & 11.54522614532995 \\
\hline $\mathrm{C}$ & -4.25137465425307 & 8.59519255547742 & 9.47272899061343 \\
\hline $\mathrm{H}$ & -4.46709505971736 & 9.24928168863843 & 8.61901106359643 \\
\hline $\mathrm{H}$ & -4.98745001341148 & 7.78032947671982 & 9.45261854915960 \\
\hline $\mathrm{C}$ & -2.82204722955437 & 8.03287118155913 & 9.34379760153004 \\
\hline $\mathrm{H}$ & -2.77193805417610 & 7.05851950951650 & 9.86221153172001 \\
\hline $\mathrm{C}$ & -2.36899017132657 & 7.88158179381403 & 7.84438629947087 \\
\hline $\mathrm{C}$ & -1.15015452108834 & 6.92807294256801 & 7.66505992698906 \\
\hline $\mathrm{C}$ & -0.29887676309092 & 7.13137539814572 & 6.56569279557274 \\
\hline $\mathrm{H}$ & -0.50574296367005 & 7.97239482930868 & 5.90219469883847 \\
\hline $\mathrm{C}$ & 0.79096877956919 & 6.29654688387751 & 6.31348472205533 \\
\hline $\mathrm{H}$ & 1.44155309388061 & 6.47991672329246 & 5.45600937915007 \\
\hline $\mathrm{C}$ & 1.04948024773455 & 5.22483895923977 & 7.17481211930087 \\
\hline $\mathrm{C}$ & 0.20552291674337 & 4.97418191488065 & 8.25721393078768 \\
\hline $\mathrm{H}$ & 0.39535545874947 & 4.12868671469919 & 8.92097426299395 \\
\hline $\mathrm{C}$ & -0.88317966505510 & 5.82384762614396 & 8.49076087158757 \\
\hline $\mathrm{H}$ & -1.52543088192492 & 5.59776636220494 & 9.34434093633718 \\
\hline $\mathrm{C}$ & -3.52991698724387 & 7.23409099348480 & 7.03331364605176 \\
\hline $\mathrm{C}$ & -4.00338240857447 & 5.94261230991513 & 7.31682230478371 \\
\hline $\mathrm{H}$ & -3.55285075377401 & 5.35180342299150 & 8.11747270517795 \\
\hline $\mathrm{C}$ & -5.04901918301018 & 5.37268180070748 & 6.58701894684947 \\
\hline
\end{tabular}




$\begin{array}{lrrr}\mathrm{H} & -5.41233656423509 & 4.37268829902008 & 6.83092442008144 \\ \mathrm{C} & -5.62508382597893 & 6.09723013056092 & 5.53905436694107 \\ \mathrm{C} & -5.17122740896267 & 7.38067065068941 & 5.23323664242497 \\ \mathrm{H} & -5.63006793937309 & 7.94465116644820 & 4.41885126476371 \\ \mathrm{C} & -4.13235311616938 & 7.94154370325136 & 5.98483328970997 \\ \mathrm{H} & -3.77763327829933 & 8.94864361278806 & 5.76512122999060 \\ \mathrm{~N} & -0.12927467238321 & 11.64914899284981 & 6.70523673792535 \\ \mathrm{~N} & 0.34548613162024 & 9.64321773996093 & 8.63886387226109 \\ \mathrm{~N} & -1.99276732827259 & 9.02274485528116 & 10.10428925422039 \\ \mathrm{O} & -0.80063127784808 & 12.11167705041748 & 9.34577880317703 \\ \mathrm{O} & -2.09516616894922 & 9.14875864727107 & 7.33209727237414\end{array}$

$\mathrm{Fe}^{\mathrm{III}}(\mathrm{BDPP})\left(\mathrm{O}_{2}{ }^{\circ}\right)(3)(S=3)$ :

$\begin{array}{lrrr}\mathrm{Fe} & 1.951648 & 3.352647 & 18.365824 \\ \mathrm{O} & 0.285517 & 4.462169 & 18.745998 \\ \mathrm{O} & -0.876834 & 4.759570 & 19.116170 \\ \mathrm{O} & 2.833465 & 4.863863 & 17.547830 \\ \mathrm{O} & 1.052787 & 1.817025 & 19.106950 \\ \mathrm{~N} & 1.653766 & 2.798412 & 16.170781 \\ \mathrm{~N} & 3.669333 & 2.136842 & 17.951469 \\ \mathrm{~N} & 3.020375 & 3.255953 & 20.393969 \\ \mathrm{C} & 3.042481 & 4.906006 & 16.174129 \\ \mathrm{C} & 2.997345 & 6.366250 & 15.668693 \\ \mathrm{C} & 2.936758 & 7.410335 & 16.596526 \\ \mathrm{H} & 2.896584 & 7.152372 & 17.655029 \\ \mathrm{C} & 2.919853 & 8.742954 & 16.171503 \\ \mathrm{H} & 2.876557 & 9.546315 & 16.911940 \\ \mathrm{C} & 2.952216 & 9.051074 & 14.809845 \\ \mathrm{H} & 2.936610 & 10.091720 & 14.474851 \\ \mathrm{C} & 3.003469 & 8.013182 & 13.874669 \\ \mathrm{H} & 3.027005 & 8.240881 & 12.805231 \\ \mathrm{C} & 3.032844 & 6.684384 & 14.302147 \\ \mathrm{H} & 3.088284 & 5.893503 & 13.551646 \\ \mathrm{C} & 4.454263 & 4.384669 & 15.804342\end{array}$




\begin{tabular}{|c|c|c|c|}
\hline $\mathrm{C}$ & 4.795061 & 3.908142 & 14.529605 \\
\hline $\mathrm{H}$ & 4.042074 & 3.845946 & 13.742822 \\
\hline $\mathrm{C}$ & 6.100729 & 3.504588 & 14.233239 \\
\hline $\mathrm{H}$ & 6.340118 & 3.126322 & 13.235452 \\
\hline $\mathrm{C}$ & 7.097121 & 3.577918 & 15.207811 \\
\hline $\mathrm{H}$ & 8.116343 & 3.257351 & 14.976144 \\
\hline C & 6.773102 & 4.066065 & 16.478105 \\
\hline $\mathrm{H}$ & 7.545431 & 4.138159 & 17.248550 \\
\hline C & 5.467992 & 4.464441 & 16.768845 \\
\hline $\mathrm{H}$ & 5.201173 & 4.849526 & 17.753066 \\
\hline C & 1.897733 & 4.097529 & 15.479969 \\
\hline $\mathrm{H}$ & 2.140652 & 3.889842 & 14.423606 \\
\hline C & 0.540784 & 4.819309 & 15.562749 \\
\hline $\mathrm{H}$ & 0.550594 & 5.520788 & 16.405916 \\
\hline $\mathrm{H}$ & 0.370903 & 5.411265 & 14.654557 \\
\hline C & -0.525014 & 3.722580 & 15.777061 \\
\hline $\mathrm{H}$ & -1.071251 & 3.895062 & 16.712317 \\
\hline $\mathrm{H}$ & -1.267050 & 3.691865 & 14.965672 \\
\hline $\mathrm{C}$ & 0.260156 & 2.411271 & 15.839734 \\
\hline $\mathrm{H}$ & -0.104990 & 1.710130 & 16.604147 \\
\hline $\mathrm{H}$ & 0.247036 & 1.898581 & 14.858410 \\
\hline $\mathrm{C}$ & 2.582778 & 1.703648 & 15.849439 \\
\hline $\mathrm{H}$ & 2.030040 & 0.761840 & 15.998436 \\
\hline $\mathrm{H}$ & 2.891602 & 1.733006 & 14.789673 \\
\hline $\mathrm{C}$ & 3.794725 & 1.605775 & 16.740032 \\
\hline $\mathrm{C}$ & 4.942230 & 0.897190 & 16.386978 \\
\hline $\mathrm{H}$ & 5.059221 & 0.495760 & 15.379724 \\
\hline $\mathrm{C}$ & 5.925482 & 0.708761 & 17.357336 \\
\hline $\mathrm{H}$ & 6.825974 & 0.139606 & 17.116403 \\
\hline $\mathrm{C}$ & 5.751541 & 1.244894 & 18.633604 \\
\hline $\mathrm{H}$ & 6.498195 & 1.093230 & 19.415130 \\
\hline $\mathrm{C}$ & 4.601447 & 1.994393 & 18.885812 \\
\hline $\mathrm{C}$ & 4.392502 & 2.768175 & 20.167098 \\
\hline $\mathrm{H}$ & 5.040078 & 3.656274 & 20.092044 \\
\hline $\mathrm{H}$ & 4.757724 & 2.185440 & 21.030630 \\
\hline
\end{tabular}




\begin{tabular}{|c|c|c|c|}
\hline $\mathrm{C}$ & 3.055264 & 4.540085 & 21.137136 \\
\hline $\mathrm{H}$ & 3.417279 & 5.334204 & 20.467785 \\
\hline $\mathrm{H}$ & 3.758516 & 4.451924 & 21.988932 \\
\hline $\mathrm{C}$ & 1.628745 & 4.749664 & 21.636718 \\
\hline $\mathrm{H}$ & 1.055628 & 5.350918 & 20.920507 \\
\hline $\mathrm{H}$ & 1.618684 & 5.281096 & 22.598995 \\
\hline $\mathrm{C}$ & 1.038065 & 3.327979 & 21.735414 \\
\hline $\mathrm{H}$ & 0.775763 & 3.045185 & 22.763629 \\
\hline $\mathrm{H}$ & 0.121751 & 3.257080 & 21.141224 \\
\hline $\mathrm{C}$ & 2.109070 & 2.364306 & 21.182042 \\
\hline $\mathrm{H}$ & 2.682118 & 1.934540 & 22.021312 \\
\hline $\mathrm{C}$ & 1.487167 & 1.234272 & 20.289767 \\
\hline $\mathrm{C}$ & 2.475360 & 0.081993 & 19.998586 \\
\hline $\mathrm{C}$ & 2.332752 & -0.604720 & 18.783724 \\
\hline $\mathrm{H}$ & 1.542683 & -0.279014 & 18.106028 \\
\hline $\mathrm{C}$ & 3.181924 & -1.656780 & 18.444243 \\
\hline $\mathrm{H}$ & 3.053287 & -2.170806 & 17.487793 \\
\hline $\mathrm{C}$ & 4.195135 & -2.058416 & 19.322282 \\
\hline $\mathrm{H}$ & 4.865068 & -2.879456 & 19.054820 \\
\hline $\mathrm{C}$ & 4.331964 & -1.403157 & 20.546736 \\
\hline $\mathrm{H}$ & 5.108321 & -1.711114 & 21.252251 \\
\hline $\mathrm{C}$ & 3.475098 & -0.348176 & 20.881859 \\
\hline $\mathrm{H}$ & 3.607946 & 0.141041 & 21.847862 \\
\hline $\mathrm{C}$ & 0.281219 & 0.630910 & 21.046256 \\
\hline $\mathrm{C}$ & 0.435500 & -0.184631 & 22.175498 \\
\hline $\mathrm{H}$ & 1.432901 & -0.452694 & 22.528199 \\
\hline $\mathrm{C}$ & -0.679293 & -0.685683 & 22.852910 \\
\hline $\mathrm{H}$ & -0.536486 & -1.323716 & 23.729311 \\
\hline $\mathrm{C}$ & -1.968730 & -0.374115 & 22.411408 \\
\hline $\mathrm{H}$ & -2.839526 & -0.767652 & 22.94174 \\
\hline $\mathrm{C}$ & -2.130114 & 0.439420 & 21.285979 \\
\hline $\mathrm{H}$ & -3.131925 & 0.697205 & 20.93061 \\
\hline $\mathrm{C}$ & -1.013508 & 0.934236 & 20.607039 \\
\hline $\mathrm{H}$ & -1.122109 & 1.566035 & 19.72503 \\
\hline
\end{tabular}


$\mathrm{Fe}^{\mathrm{III}}(\mathrm{BDPP})\left(\mathrm{O}_{2}{ }^{\circ}\right)(S=2)$ :

$\begin{array}{lrrr}\mathrm{Fe} & 1.909703 & 3.383413 & 18.376237 \\ \mathrm{O} & 0.365561 & 4.489157 & 18.699610 \\ \mathrm{O} & -0.775402 & 4.154883 & 19.184275 \\ \mathrm{O} & 2.855915 & 4.850910 & 17.555327 \\ \mathrm{O} & 1.064975 & 1.824708 & 19.099184 \\ \mathrm{~N} & 1.624922 & 2.818869 & 16.190800 \\ \mathrm{~N} & 3.635978 & 2.161124 & 17.956186 \\ \mathrm{~N} & 2.994223 & 3.289285 & 20.382581 \\ \mathrm{C} & 3.057850 & 4.895736 & 16.179487 \\ \mathrm{C} & 3.028399 & 6.358998 & 15.681632 \\ \mathrm{C} & 2.955700 & 7.400104 & 16.611682 \\ \mathrm{H} & 2.896735 & 7.139749 & 17.668638 \\ \mathrm{C} & 2.949081 & 8.733844 & 16.189768 \\ \mathrm{H} & 2.896020 & 9.535270 & 16.931631 \\ \mathrm{C} & 3.003313 & 9.045495 & 14.829583 \\ \mathrm{H} & 2.995790 & 10.087052 & 14.497154 \\ \mathrm{C} & 3.066519 & 8.010149 & 13.892279 \\ \mathrm{H} & 3.107961 & 8.240761 & 12.824021 \\ \mathrm{C} & 3.086481 & 6.680305 & 14.316728 \\ \mathrm{H} & 3.153008 & 5.890655 & 13.565919 \\ \mathrm{H} & 4.134642 & 3.886594 & 14.437121 \\ \mathrm{C} & & & \end{array}$




\begin{tabular}{|c|c|c|c|}
\hline $\mathrm{C}$ & 0.552013 & 4.858120 & 15.564311 \\
\hline $\mathrm{H}$ & 0.571297 & 5.563782 & 16.403378 \\
\hline $\mathrm{H}$ & 0.397664 & 5.445519 & 14.650277 \\
\hline $\mathrm{C}$ & -0.535150 & 3.783476 & 15.784442 \\
\hline $\mathrm{H}$ & -1.076674 & 3.968451 & 16.720033 \\
\hline $\mathrm{H}$ & -1.275500 & 3.760239 & 14.971228 \\
\hline $\mathrm{C}$ & 0.223178 & 2.457556 & 15.860604 \\
\hline $\mathrm{H}$ & -0.157561 & 1.771430 & 16.630926 \\
\hline $\mathrm{H}$ & 0.204900 & 1.935079 & 14.884896 \\
\hline $\mathrm{C}$ & 2.532405 & 1.705075 & 15.873799 \\
\hline $\mathrm{H}$ & 1.967993 & 0.774067 & 16.043928 \\
\hline $\mathrm{H}$ & 2.827492 & 1.716177 & 14.809946 \\
\hline $\mathrm{C}$ & 3.751115 & 1.611064 & 16.752705 \\
\hline $\mathrm{C}$ & 4.894203 & 0.895293 & 16.400344 \\
\hline $\mathrm{H}$ & 5.001310 & 0.478532 & 15.398320 \\
\hline $\mathrm{C}$ & 5.886942 & 0.722309 & 17.363638 \\
\hline $\mathrm{H}$ & 6.784936 & 0.149119 & 17.123241 \\
\hline $\mathrm{C}$ & 5.725277 & 1.280048 & 18.632230 \\
\hline $\mathrm{H}$ & 6.479040 & 1.141520 & 19.409309 \\
\hline $\mathrm{C}$ & 4.577521 & 2.032918 & 18.882970 \\
\hline $\mathrm{C}$ & 4.374404 & 2.826839 & 20.150752 \\
\hline $\mathrm{H}$ & 5.001652 & 3.726626 & 20.049876 \\
\hline $\mathrm{H}$ & 4.758331 & 2.267881 & 21.021797 \\
\hline $\mathrm{C}$ & 3.006639 & 4.578652 & 21.119923 \\
\hline $\mathrm{H}$ & 3.339640 & 5.376598 & 20.440144 \\
\hline $\mathrm{H}$ & 3.725146 & 4.511091 & 21.960286 \\
\hline $\mathrm{C}$ & 1.583243 & 4.755027 & 21.639440 \\
\hline $\mathrm{H}$ & 0.985005 & 5.332924 & 20.924514 \\
\hline $\mathrm{H}$ & 1.573450 & 5.293312 & 22.597790 \\
\hline $\mathrm{C}$ & 1.028515 & 3.320278 & 21.754222 \\
\hline $\mathrm{H}$ & 0.796101 & 3.033274 & 22.788421 \\
\hline $\mathrm{H}$ & 0.102146 & 3.237236 & 21.178057 \\
\hline $\mathrm{C}$ & 2.107280 & 2.378690 & 21.179253 \\
\hline $\mathrm{H}$ & 2.701095 & 1.953579 & 22.005685 \\
\hline $\mathrm{C}$ & 1.499398 & 1.242843 & 20.285375 \\
\hline
\end{tabular}




$\begin{array}{lrrr}\mathrm{C} & 2.496316 & 0.097079 & 19.999847 \\ \mathrm{C} & 2.350764 & -0.605683 & 18.794396 \\ \mathrm{H} & 1.554673 & -0.293946 & 18.117198 \\ \mathrm{C} & 3.202836 & -1.658084 & 18.463693 \\ \mathrm{H} & 3.071792 & -2.184479 & 17.514337 \\ \mathrm{C} & 4.221305 & -2.045072 & 19.342381 \\ \mathrm{H} & 4.892561 & -2.867430 & 19.082480 \\ \mathrm{C} & 4.361469 & -1.373626 & 20.557605 \\ \mathrm{H} & 5.141820 & -1.670082 & 21.263589 \\ \mathrm{C} & 3.502485 & -0.317337 & 20.883231 \\ \mathrm{H} & 3.637635 & 0.183907 & 21.842728 \\ \mathrm{C} & 0.290294 & 0.628915 & 21.029212 \\ \mathrm{C} & 0.445036 & -0.210528 & 22.140753 \\ \mathrm{H} & 1.442463 & -0.486426 & 22.487161 \\ \mathrm{C} & -0.669547 & -0.724859 & 22.808882 \\ \mathrm{H} & -0.526039 & -1.380910 & 23.671841 \\ \mathrm{C} & -1.958927 & -0.404069 & 22.374225 \\ \mathrm{H} & -2.829803 & -0.808474 & 22.896323 \\ \mathrm{C} & -2.120158 & 0.434090 & 21.266907 \\ \mathrm{H} & -3.121594 & 0.701590 & 20.917752 \\ \mathrm{C} & -1.004508 & 0.943683 & 20.597569 \\ \mathrm{H} & -1.117489 & 1.600593 & 19.735024\end{array}$

$\mathrm{Co}^{\mathrm{III}}(\mathrm{BDPP})\left(\mathrm{O}_{2}{ }^{\circ}\right)(4)$ :

$\begin{array}{lrrr}\text { Co } & -0.077080 & -0.177359 & 0.993453 \\ \mathrm{O} & -0.258990 & -0.663318 & 2.825244 \\ \mathrm{O} & 0.231315 & 0.093113 & 3.758649 \\ \mathrm{O} & 0.078293 & 1.674319 & 1.353370 \\ \mathrm{O} & -0.272041 & -2.020381 & 0.611320 \\ \mathrm{~N} & -2.045306 & 0.158401 & 0.719370 \\ \mathrm{~N} & 0.049713 & 0.153122 & -0.875805 \\ \mathrm{~N} & 1.911880 & -0.452879 & 0.870809 \\ \mathrm{C} & -1.027034 & 0.498075 & -2.939850 \\ \mathrm{C} & -1.075789 & 0.261718 & -1.568966\end{array}$




\begin{tabular}{|c|c|c|c|}
\hline $\mathrm{C}$ & -2.299792 & 0.006411 & -0.741522 \\
\hline $\mathrm{C}$ & -2.968079 & -0.754285 & 1.458675 \\
\hline $\mathrm{C}$ & -3.137321 & -0.168425 & 2.860228 \\
\hline $\mathrm{C}$ & -2.615563 & 1.280236 & 2.762915 \\
\hline $\mathrm{C}$ & -2.299512 & 1.537725 & 1.281785 \\
\hline $\mathrm{C}$ & -2.018398 & 4.701516 & 1.835953 \\
\hline $\mathrm{C}$ & -2.072141 & 5.769419 & 2.736160 \\
\hline $\mathrm{C}$ & -1.218165 & 5.797305 & 3.844148 \\
\hline $\mathrm{C}$ & -0.320714 & 4.744283 & 4.045219 \\
\hline $\mathrm{C}$ & -0.273970 & 3.672399 & 3.147912 \\
\hline $\mathrm{C}$ & -1.119333 & 3.643014 & 2.031497 \\
\hline $\mathrm{C}$ & -2.072400 & 3.311356 & -1.183422 \\
\hline $\mathrm{C}$ & -1.911451 & 3.887715 & -2.448854 \\
\hline $\mathrm{C}$ & -0.637357 & 4.206072 & -2.919519 \\
\hline $\mathrm{C}$ & 0.473364 & 3.954795 & -2.106519 \\
\hline $\mathrm{C}$ & 0.306002 & 3.381221 & -0.847160 \\
\hline $\mathrm{C}$ & -0.966518 & 3.034074 & -0.368043 \\
\hline $\mathrm{C}$ & -1.047054 & 2.438184 & 1.060376 \\
\hline $\mathrm{C}$ & 0.227444 & 0.586037 & -3.548015 \\
\hline $\mathrm{C}$ & 1.390635 & 0.445190 & -2.787853 \\
\hline $\mathrm{C}$ & 1.256681 & 0.236266 & -1.418835 \\
\hline $\mathrm{C}$ & 2.356138 & 0.182643 & -0.401260 \\
\hline $\mathrm{C}$ & 2.668343 & 0.133956 & 2.035445 \\
\hline $\mathrm{C}$ & 3.094036 & -1.040969 & 2.920565 \\
\hline $\mathrm{C}$ & 2.294273 & -2.241512 & 2.408708 \\
\hline $\mathrm{C}$ & 2.106140 & -1.955461 & 0.920723 \\
\hline $\mathrm{C}$ & 1.753827 & -5.120935 & 0.427691 \\
\hline $\mathrm{C}$ & 1.689050 & -6.406919 & 0.970038 \\
\hline $\mathrm{C}$ & 0.698522 & -6.727022 & 1.904817 \\
\hline $\mathrm{C}$ & -0.219133 & -5.748385 & 2.296256 \\
\hline $\mathrm{C}$ & -0.147967 & -4.460343 & 1.757300 \\
\hline $\mathrm{C}$ & 0.836478 & -4.133365 & 0.816617 \\
\hline $\mathrm{C}$ & 2.245045 & -2.874162 & -1.972223 \\
\hline $\mathrm{C}$ & 2.277359 & -3.022655 & -3.363558 \\
\hline $\mathrm{C}$ & 1.088383 & -3.114240 & -4.087316 \\
\hline
\end{tabular}




$\begin{array}{lrrr}\mathrm{C} & -0.132188 & -3.065853 & -3.404858 \\ \mathrm{C} & -0.157185 & -2.916869 & -2.019181 \\ \mathrm{C} & 1.028859 & -2.804197 & -1.277907 \\ \mathrm{C} & 0.896127 & -2.689463 & 0.262207 \\ \mathrm{H} & -1.944984 & 0.611657 & -3.517540 \\ \mathrm{H} & -2.575937 & -1.045427 & -0.905548 \\ \mathrm{H} & -3.150784 & 0.629479 & -1.058651 \\ \mathrm{H} & -3.935408 & -0.764591 & 0.926515 \\ \mathrm{H} & -2.534163 & -1.761571 & 1.430604 \\ \mathrm{H} & -4.192881 & -0.215113 & 3.166958 \\ \mathrm{H} & -2.548948 & -0.735646 & 3.590016 \\ \mathrm{H} & -3.337759 & 2.022058 & 3.127366 \\ \mathrm{H} & -1.704361 & 1.392765 & 3.357224 \\ \mathrm{H} & -3.192894 & 1.944081 & 0.781375 \\ \mathrm{H} & -2.671487 & 4.710510 & 0.961404 \\ \mathrm{H} & -2.775134 & 6.588976 & 2.560871 \\ \mathrm{H} & -1.249429 & 6.637883 & 4.543177 \\ \mathrm{H} & 0.345218 & 4.751842 & 4.912726 \\ \mathrm{H} & 0.405171 & 2.832740 & 3.297710 \\ \mathrm{H} & -3.082415 & 3.081797 & -0.840790 \\ \mathrm{H} & -2.790306 & 4.095896 & -3.064732 \\ \mathrm{H} & -0.508451 & 4.660272 & -3.905043 \\ \mathrm{H} & 1.474739 & 4.217039 & -2.459677 \\ \mathrm{H} & 1.160917 & 3.186191 & -0.198020 \\ \mathrm{H} & 0.300512 & 0.767580 & -4.622339 \\ \mathrm{H} & 2.377449 & 0.500495 & -3.248944 \\ \mathrm{H} & 2.622678 & 1.222591 & -0.166717 \\ \mathrm{H} & 3.254972 & -0.317153 & -0.795133 \\ \mathrm{H} & 3.536333 & 0.690365 & 1.653816 \\ \mathrm{H} & 1.996805 & 0.834895 & 2.545026 \\ \mathrm{H} & 4.175123 & -1.226990 & 2.808452 \\ \mathrm{H} & 2.890453 & -0.835621 & 3.980220 \\ \mathrm{H} & -31801707 & -2.302976 & 2.901074 \\ \mathrm{H} & -2.188242 & 0.397801\end{array}$




$\begin{array}{lrrr}\mathrm{H} & 2.518641 & -4.894414 & -0.317914 \\ \mathrm{H} & 2.409504 & -7.165734 & 0.652668 \\ \mathrm{H} & 0.640582 & -7.734002 & 2.326067 \\ \mathrm{H} & -0.993565 & -5.988359 & 3.030075 \\ \mathrm{H} & -0.843165 & -3.677578 & 2.059632 \\ \mathrm{H} & 3.193321 & -2.815657 & -1.436063 \\ \mathrm{H} & 3.239202 & -3.073881 & -3.881112 \\ \mathrm{H} & 1.110989 & -3.239610 & -5.172953 \\ \mathrm{H} & -1.070497 & -3.153870 & -3.958976 \\ \mathrm{H} & -1.101134 & -2.884801 & -1.473712\end{array}$




\section{References}

1. Aasa, R.; Vänngård, T. EPR Signal Intensity and Powder Shapes: A Reexamination. J. Magn. Reson. 1975, 19 (3), 308-315.

2. VanAtta, R. B.; Strouse, C. E.; Hanson, L. K.; Valentine, J. S.

Peroxo(tetraphenylporphinato)manganese(III) and chloro(tetraphenylporphinato)manganese(II)

Anions. Synthesis, crystal Structures, and electronic Structures. J. Am. Chem. Soc. 1987, 109, 1425-1434.

3. Brown, P. M.; Caradoc-Davies, T. T.; Dickson, J. M. J.; Cooper, G. J. S.; Loomes, K. M.; Baker, E. N. Crystal Structure of a Substrate Complex of Myo-inositol Oxygenase, a Di-Iron Oxygenase with a Key Role in Inositol Metabolism. Proc. Natl. Acad. Sci. U. S. A. 2006, 103, 15032-15037.

4. Singh, U. P.; Sharma, A. K.; Hikichi, S.; Komatsuzaki, H.; Moro-oka, Y.; Akita, M. Hydrogen Bonding Interaction between Imidazolyl N-H Group and Peroxide: Stabilization of Mn(III)Peroxo Complex $\operatorname{TpiPr}_{2} \mathrm{Mn}\left(\eta^{2}-\mathrm{O}_{2}\right)(\mathrm{imMeH})(\mathrm{imMeH}=2$-methylimidazole). Inorg. Chim. Acta 2006, 359, 4407-4411.

5. Seo, M. S.; Kim, J. Y.; Annaraj, J.; Kim, Y.; Lee, Y.-M.; Kim, S.-J.; Kim, J.; Nam, W. $\left[\mathrm{Mn}(\mathrm{tmc})\left(\mathrm{O}_{2}\right)\right]^{+}$: A Side-On Peroxido Manganese(III) Complex Bearing a Non-Heme Ligand. Angew. Chem. Int. Ed. 2007, 46, 377-380.

6. Annaraj, J.; Cho, J.; Lee, Y. M.; Kim, S. Y.; Latifi, R.; de Visser, S. P.; Nam, W. Structural Characterization and Remarkable Axial Ligand Effect on the Nucleophilic Reactivity of a Nonheme Manganese(III)-Peroxo Complex. Angew. Chem. Int. Ed. 2009, 48, 4150-4153.

7. Kang, H.; Cho, J.; Cho, K. B.; Nomura, T.; Ogura, T.; Nam, W. Mononuclear Manganese-Peroxo and Bis(mu-oxo)dimanganese Complexes Bearing a Common N-Methylated Macrocyclic Ligand. Chem. Eur. J. 2013, 19, 14119-14125.

8. Coggins, M. K.; Kovacs, J. A. Structural and Spectroscopic Characterization of Metastable Thiolate-Ligated Manganese(III)-Alkylperoxo Species. J. Am. Chem. Soc. 2011, 133, 1247012473.

9. Coggins, M. K.; Martin-Diaconescu, V.; DeBeer, S.; Kovacs, J. A. Correlation Between Structural, Spectroscopic, and Reactivity Properties Within a Series of Structurally Analogous Metastable Manganese(III)-Alkylperoxo Complexes. J. Am. Chem. Soc. 2013, 135, 4260-4272.

10. Coggins, M. K.; Sun, X.; Kwak, Y.; Solomon, E. I.; Rybak-Akimova, E.; Kovacs, J. A. Characterization of Metastable Intermediates Formed in the Reaction Between a Mn(II) Complex and Dioxygen, Including a Crystallographic Structure of a Binuclear Mn(III)-Peroxo Species. $J$. Am. Chem. Soc. 2013, 135, 5631-5640. 
11. Coggins, M. K.; Brines, L. M.; Kovacs, J. A. Synthesis and Structural Characterization of a Series of $\mathrm{Mn}^{\mathrm{III}} \mathrm{OR}$ Complexes, Including a Water-Soluble $\mathrm{Mn}^{\mathrm{III}} \mathrm{OH}$ That Promotes Aerobic HydrogenAtom Transfer. Inorg. Chem. 2013, 52, 12383-12393.

12. Schatz, M.; Raab, V.; Foxon, S. P.; Brehm, G.; Schneider, S.; Reiher, M.; Holthausen, M. C.; Sundermeyer, J.; Schindler, S. Combined Spectroscopic and Theoretical Evidence For a Persistent End-On Copper Superoxo Complex. Angew. Chem. Int. Ed. 2004, 43, 4360-4363.

13. Cho, J.; Woo, J.; Nam, W. An "End-On" Chromium(III)-Superoxo Complex: Crystallographic and Spectroscopic Characterization and Reactivity in $\mathrm{C}-\mathrm{H}$ Bond Activation of Hydrocarbons. J. Am. Chem. Soc. 2010, 132, 5958-5959.

14. Hong, S.; Sutherlin, K. D.; Park, J.; Kwon, E.; Siegler, M. A.; Solomon, E. I.; Nam, W. Crystallographic and Spectroscopic Characterization and Reactivities of a Mononuclear Non-Haem Iron(III)-Superoxo complex. Nat. Commun. 2014, 5, 5440-5447.

15. Oddon, F.; Chiba, Y.; Nakazawa, J.; Ohta, T.; Ogura, T.; Hikichi, S. Characterization of Mononuclear Non-heme Iron(III)-Superoxo Complex with a Five-Azole Ligand Set. Angew. Chem. Int. Ed. 2015, 54, 7336-7339.

16. Stout, H. D.; Kleespies, S. T.; Chiang, C. W.; Lee, W. Z.; Que, L., Jr.; Munck, E.; Bominaar, E. L., Spectroscopic and Theoretical Study of Spin-Dependent Electron Transfer in an Iron(III) Superoxo Complex. Inorg. Chem. 2016, 55, 5215-5226. 